\title{
Cerebral Compensation during Motor Function in Friedreich Ataxia: The IMAGE- FRDA Study
}

Ian H. Harding, $\mathrm{PhD}^{1}$, Louise A. Corben, $\mathrm{PhD}^{1,2}$, Martin B. Delatycki, $\mathrm{PhD}^{1,2,3}$, Monique R. Stagnitti, BSc(Hons) ${ }^{1}$, Elsdon Storey, $\mathrm{PhD}^{4}$, Gary F. Egan, $\mathrm{PhD}^{1,5}$, Nellie GeorgiouKaristianis, $\mathrm{PhD}^{1}$

${ }^{1}$ School of Psychological Sciences \& Monash Institute of Cognitive and Clinical Neurosciences, Monash University, Melbourne, Australia ${ }^{2}$ Bruce Lefroy Centre, Murdoch Childrens Research Institute, Melbourne, Australia ${ }^{3}$ Clinical Genetics, Austin Health, Melbourne, Australia ${ }^{4}$ Department of Medicine, Monash University, Melbourne, Australia ${ }^{5}$ Monash Biomedical Imaging, Monash University, Melbourne, Australia

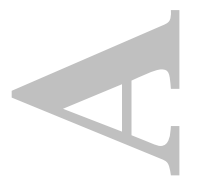

Correspondence:

Dr. Ian Harding

School of Psychological Sciences

18 Innovation Walk

Monash University, VIC, 3800

Australia

Email: ian.harding@monash.edu

Phone: +61-3-9905-9283

Fax: +61-3-9905-3948

Running Title: Compensation in Friedreich Ataxia

Word Count: 3783; Figures: 4; Tables: 1; Supplementary Materials: 2 Figures, 4 Tables

Disclosures: The authors report no conflicts of interest

Funding: Australian National Health and Medical Research Council (Project Grant 1046037; Fellowship 1106533)

Keywords: Friedreich ataxia, compensation, fMRI, motor function

This is the author manuscript accepted for publication and has undergone full peer review but has not been through the copyediting, typesetting, pagination and proofreading process, which may lead to differences between this version and the Version record. Please cite this article as doi:10.1002/ mds.27023. 


\section{Abstract}

Background: Friedreich ataxia is characterized by progressive motor incoordination that is linked to peripheral, spinal, and cerebellar neuropathy. Cerebral abnormalities are also reported in Friedreich ataxia, but their role in disease expression remains unclear.

Methods: In this cross-sectional functional magnetic resonance imaging study, 25 individuals with Friedreich ataxia and 33 healthy controls performed simple (self-paced single-finger) and complex (visually-cued multi-finger) tapping tasks to respectively gauge basic and attentionally-demanding motor behaviour. For each task, whole brain functional activations were compared between groups, and correlated with disease severity and offline measures of motor dexterity.

Results: During simple finger tapping, cerebral hyperactivation in individuals with Friedreich ataxia at the lower end of clinical severity and cerebral hypoactivation in those more severely affected was observed in premotor/ventral attention brain regions, including the supplementary motor area and anterior insula. Greater activation in this network correlated with greater offline finger tapping precision. Complex, attentionally-demanding finger tapping was also associated with cerebral hyperactivation, but in this case within dorsolateral prefrontal regions of the executive control network and superior parietal regions of the dorsal attention system. Greater offline motor precision was associated with less activation in the dorsal attention network.

Discussion: Compensatory activity is evident in the cerebral cortex in individuals with Friedreich ataxia. Early compensation followed by later decline in premotor/ventral attention systems demonstrates capacity-limited neural reserve, while the additional engagement of higher-order brain networks is indicative of compensatory task strategies. Network-level changes in cerebral brain function thus potentially serve to mitigate the impact of motor impairments in Friedreich ataxia. 


\section{Introduction}

Friedreich ataxia (FRDA), the most common inherited ataxia, is an autosomal recessive disorder defined by progressive movement incoordination. The neurological component of FRDA includes degeneration of the dorsal root ganglia, dorsal spinal tracts, and dentate nuclei

of the cerebellum ${ }^{1}$. Disrupted functional and structural connectivity between the cerebellum and cerebrum have also been reported ${ }^{2,3}$, as have functional and structural abnormalities in the cerebellar cortex, cerebral cortex, and subcortical extrapyramidal system ${ }^{4-8}$. The emerging picture of the central neuropathology underlying FRDA is thus now expanding beyond conventional focus on sub-tentorial disruptions; however, the role of the cerebrum in disease expression is not well understood.

Motor deficits are the most salient clinical features of FRDA, and include limb and gait ataxia; poor balance control; dysmetria, dysdiadochokinesia, and intention tremor; and dysarthria and dysphagia ${ }^{9}$. The neurofunctional correlates of motor dysfunction have been investigated in vivo using functional magnetic resonance imaging (fMRI) in small cohorts of between 7 and 11 individuals with FRDA ${ }^{10,11}$. These studies found consistent evidence of functional deficits in cerebellar lobules V-VI, the supplementary motor areas, and the inferior parietal lobules during various finger and hand tapping tasks. Other frontal, prefrontal, posterior parietal and cerebellar brain regions were more inconsistently implicated, and observations included both hypoactivation and hyperactivation relative to healthy controls $^{10,11}$. This pattern of findings suggests that a common set of brain abnormalities may contribute to the motor deficits in FRDA, while task-specific mechanisms of functional compensation $^{12}$ or down-stream consequence (i.e., diaschisis ${ }^{13}$ ) may operate elsewhere. 
The current study had two primary aims. First, we sought to replicate and extend upon investigations of simple motor functions in $\mathrm{FRDA}^{10,11}$ using a larger research cohort, providing for increased statistical power and more reliable generalizability of results.

Additionally, by recruiting individuals across a wide range of clinical severity, we sought to cross-sectionally investigate links between disease severity and detrimental or compensatory functional changes in the brain.

Second, we aimed to investigate complex motor-related function in FRDA by employing an attentionally demanding motor paradigm. Adaptive real-world interactions rely not just on the ability to produce motor responses, but to do so in a goal-oriented and contextually relevant fashion. Behavioral studies of FRDA indicate deficits not only in motor execution, but also in motor planning and adaptation of motor output in response to changing contextual conditions ${ }^{14,15}$. These results indicate that neural abnormalities may manifest at the interface between cognitive and motor processes within the cerebral cortex ${ }^{16}$. Moreover, greater task demands may also emphasize underlying functional brain deficits, or challenge the limits of active compensatory processes ${ }^{17}$. Taken together, investigating attentionally demanding motor production may more closely approximate real-world behavioral demands and provide new insights into the role the cerebrum may play in the morbidity of FRDA.

In both task manipulations, we predicted that functional hypoactivation would manifest in core motor-related brain regions that have previously been associated with FRDA, including lobules V-VI of the cerebellum, the supplementary motor areas, and the inferior parietal lobules. Compensatory hyperactivation was expected in alternate networks, encompassing the premotor and prefrontal cortices, which are engaged to support neural deficiencies in primary task $\operatorname{areas}^{17,18}$. 


\section{Materials and Methods}

\section{Participants}

Data from 25 individuals with FRDA, homozygous for a GAA expansion in intron 1 of $F X N$, and 33 age and gender-matched control participants without neurological symptoms were available for analysis (Table 1). A further 8 FRDA and 4 control datasets were excluded after data collection due to in-scanner motion or sleep, incorrect task performance, or data

corruption (see Supplementary Materials). Six individuals met criteria for late-onset FRDA ( $>25$ years old); supplementary analyses showed that between-group differences were not driven or suppressed by this subgroup (Table S4). All participants were right-handed with the

exception of 1 FRDA. The data was collected as part of the IMAGE-FRDA study ${ }^{4-6}$ (see Supplementary Materials). This project is sanctioned by the Monash Health Human Research Ethics Committee; all participants gave informed, written consent.

\section{$<<$ TABLE $1>>$}

$\sqrt{1}$

\section{Offline Motor Tasks}

Participants performed speeded and paced finger tapping tasks ${ }^{19}$. During speeded tapping, participants pressed a button with their non-dominant index finger as rapidly as possible for 10s, followed by an approximate 10s rest period, repeated five times (Figure S1A). Average tapping rate (Hz) interval was recorded (Table 1). During paced tapping, participants alternated between pressing two buttons, one with each thumb, in time with a repeated tone presented every 550ms $(1.8 \mathrm{~Hz})$. After 11 taps, the tone ceased and participants were required to maintain the same pace for an additional 31 taps. This procedure was repeated five times over approximately 3mins (Figure S1B). Tapping precision was calculated as the inverse of 
the standard deviation of the difference between observed and correct inter-tap intervals

\section{(Table 1).}

Online Behavioral Stimuli and fMRI Task Design

During fMRI recording, two experimental motor paradigms were performed: a self-paced regularly-timed single-finger tapping paradigm $(\mathrm{SF})^{10}$, and a visually-cued irregularly-timed multi-finger tapping paradigm (MF); Figure S1. Each paradigm started and ended with a 16s rest period, and consisted of four $24 \mathrm{~s}$ task blocks interspersed by $16 \mathrm{~s}$ rest blocks (176s total per task). During each task, an image of the palmar aspect of a right hand with fingers abducted was shown on a black background. During the SF task, a green circle appeared on the index finger continuously for the 24 s duration of the block (Figure S1C). With this cue, participants were asked to tap their index finger and thumb together at $0.67 \mathrm{~Hz}$, a rate at which they were trained immediately prior to data collection (while in the scanner). Average tapping rate was recorded. During the MF task blocks, the small green circle appeared in a random order on the index, middle, ring or little finger for $300 \mathrm{~ms}$, with a varied interstimulus interval averaging $1200 \mathrm{~ms}$ (varied at 550ms, 940ms, or 2240ms; Figure S1D). Tapping accuracy, defined as the percentage of trials in which the indicated finger was correctly tapped to the thumb, was recorded. The order of SF and MF task presentation was counterbalanced across participants.

Stimuli were presented using E-prime (Psychological Software Tools, Pittsburgh, USA). A MRI-compatible right-handed glove with movement sensors (Fifth Dimension Technology, www.5DT.com) was used to record the kinematics of the finger movements. During the prescan practice session, the kinematic characteristics (range of each finger movement) were calibrated for each individual. 


\section{MRI data acquisition and preprocessing}

All participants were scanned using a 3 Tesla Siemens Skyra scanner (Siemens, Erlangen, Germany) with a 32-channel head coil at Monash Biomedical Imaging, Victoria, Australia. The functional run consisted of 157 whole brain, gradient-echo echo-planar images (GREEPIs) comprising 44 interleaved, contiguous axial slices $\left(\mathrm{TR}=2500 \mathrm{~ms}\right.$; $\mathrm{TE}=30 \mathrm{~ms}$; flip $=90^{\circ}$; $3 \mathrm{~mm}$ isotropic voxels; FOV=192 $\times 192 \mathrm{~mm})$. A whole-brain T1-weighted magnetizationprepared rapid gradient-echo (MPRAGE) structural image was also acquired for each participant (176 sagittal slices; $1.0 \mathrm{~mm}$ isotropic voxels; TR=1900ms; TE=2.19ms; $\mathrm{FOV}=256 \times 256 \mathrm{~mm})$.

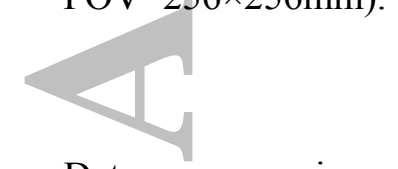

Data preprocessing using SPM12 (Functional Imaging Laboratory, UCL, UK;

www.fil.ion.ucl.ac.uk/spm/) consisted of: (i) temporal registration to the middle slice of each functional volume; (ii) spatial alignment to the first volume of the run; (iii) spatial unwarping to account for susceptibility-by-movement noise variance; (iii) coregistration with the T1weighted structural image; (iv) skull-stripping, segmentation, and non-linear normalization of the T1 (and co-registered EPIs) to standard anatomical space (MNI); and (v) spatial smoothing using a Gaussian kernel of 5mm full-width at half-maximum (FWHM).

\section{Data Analysis: Group Activation Differences}

Task fMRI effects were quantified using hierarchical general linear modeling. At the individual level, the task conditions (SF and MF) were coded as block predictors, convolved with a canonical hemodynamic response function. Additional regressors were included in the

GLM to robustly account for motion-related variance (3 planes of translation; 3 axes of rotation; and their first derivatives) and physiological noise (5 principal components of the fMRI signal in the white matter and cerebrospinal fluid, i.e., CompCor ${ }^{20}$ ). 
Contrast estimates of the individual-level SF and MF effects were entered into group-level two-sample t-tests to infer population differences across the whole brain, while controlling for age, gender, and task performance. Separate group-level models were estimated for the SF and MF task effects. Homogeneity of variance between groups was not assumed; heteroscedasticity was instead estimated using a standard ReML approach implemented in SPM12 and the statistics and degrees of freedom were adjusted as appropriate (analogous to Welch's t-test). Statistical thresholds were corrected for multiple comparisons (cluster-based family-wise error corrected $\mathrm{p}<0.05$ ) using non-parametric Monte Carlo simulations.

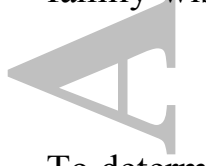

To determine whether significant between-group activation differences were associated with brain atrophy, group comparisons (two-sample t-tests) of grey matter volume (assessed by voxel-based morphometry ${ }^{5}$ ) and function-structure correlations were assessed (Supplementary Materials).

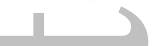

\section{Data Analysis: Clinical Correlations}

Linear relationships between task activations and disease severity across the whole brain were assessed using multiple regression models with Friedreich Ataxia Rating Scale (FARS; higher scores indicates more severe disease $\mathrm{e}^{21}$ ) score as the predictor variable and age, gender, and task performance as nuisance covariates. Regressions modeling the GAA triplet-repeat expansion on the shorter $F X N$ allele (GAA1) were also assessed, controlling for FARS score.

Further exploration and characterization of significant clinical effects was undertaken using a post-hoc median split. The FRDA sample was divided into two sub-groups based on disease 
severity $\left(\mathrm{n}_{\mathrm{low}}=12\right.$, mean $\mathrm{FARS}_{\mathrm{low}}=58 ; \mathrm{n}_{\text {high }}=13$, mean $\left.\mathrm{FARS}_{\text {high }}=95\right)$, and planned post-hoc comparisons were undertaken between each clinical sub-group and the healthy control cohort.

\section{Brain-Behaviour Correlations}

Activity within disease-sensitive functional regions (i.e., between-group differences or clinical correlations) was further assessed for correlations with offline motor performance measures. As detailed below, when overlaid onto an atlas of well-described functional brain networks $^{22,23}$, the observed task-related functional abnormalities mapped selectively onto different networks. SP and MF task-related activations were averaged across disease-sensitive

regions falling within each network, and correlated with offline motor behavior (speeded motor performance and paced motor precision).

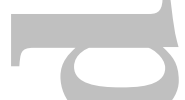

\section{Results}

\section{Finger-Tapping Behavior}

Offline maximal tapping rate and tapping precision were significantly slower in individuals with FRDA relative to controls (both $\mathrm{p}<0.001$; Table 1). Online self-paced tapping rate was significantly greater in the FRDA cohort ( $\mathrm{p}=0.022$ ), while multi-finger accuracy was matched $(\mathrm{p}=0.90$, although a majority of participants were operating at ceiling; Table 1).

\section{Self-Paced, Single Finger Brain Activations}

During self-paced single finger tapping, both cohorts engaged the left primary motor (M1) and somatosensory cortices (S1), midline supplementary motor area (SMA), right inferior parietal lobule (IPL), and right cerebellar lobule V (Figure 1; Table S1). Controls additionally showed evidence of significant activation in the right and left ventral premotor cortex, left insula, and right cerebellar lobule VIIIb. 
$<<$ FIGURE $1>>$

Relative to the control cohort, individuals with FRDA had significantly reduced activations in right lobule VI of the cerebellum (Figure 2; Table S2); no significant activation increases were evident. Functional abnormalities in lobule VI were not significantly associated with brain atrophy (Supplementary Materials). A supplementary subgroup analysis confirmed that group differences in tapping rate were not driving this effect (Supplementary Materials).

In the FRDA cohort, greater disease severity (FARS score) was associated with lesser activation in the supplementary motor area (SMA), left anterior insula (aIns), left inferior parietal lobule (IPL), and left superior temporal gyrus (STG) (Figure 2; Table S2). No significant associations with GAA1 (controlling for FARS) were evident.

As illustrated in Figure 2, post-hoc median-split comparisons revealed, relative to healthy controls, significantly increased activations in the FARS $\mathrm{S}_{\mathrm{low}}$ subgroup and decreased

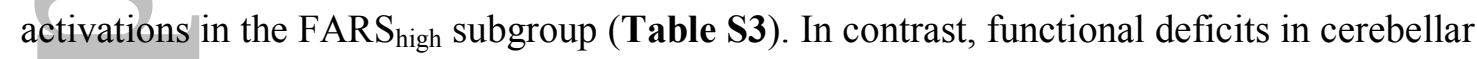
lobule VI showed monotonic decline from healthy to FARS $_{\text {low }}$ to FARS $\mathrm{Figh}_{\text {. }}$

$<<$ FIGURE 2>>

\section{Visually Cued, Multi-Finger Brain Activations}

Cued multi-finger tapping was associated with activations in both cohorts bilaterally in the motor system (M1, S1, SMA, dorsal and ventral premotor), intraparietal parietal sulci (IPS), inferior and superior parietal lobules, caudate nuclei, and cerebellar lobules V-VII and VIIbVIIIb. Additionally activation was observed in the FRDA cohort in the dorsolateral prefrontal 
cortex (dlPFC) bilaterally, the dorsal precuneus, and posterior aspects of the right middle temporal gyrus (Figure 1; Table S1).

Relative to healthy controls, individuals with FRDA elicited greater activation in bilateral dlPFC, ventrolateral premotor areas, IPS, and caudate nuclei, as well as the medial precuneus, right fusiform gyrus, and left cerebellar crus I (Figure 3; Table S2). These functional abnormalities were not significantly associated with brain atrophy (Supplementary Materials). 8

There was no evidence of FARS correlations at whole-brain corrected levels, but within the mask of regions demonstrating between-group activation differences, positive associations were found in the precuneus $\left(t_{\max }=2.71 ; \mathrm{K}_{\mathrm{E}}=88 ; \mathrm{p}_{\text {corrected }}=0.033\right)$ and right IPS $\left(t_{\max }=3.47\right.$; $\left.\mathrm{K}_{\mathrm{E}}=94 ; \mathrm{p}_{\text {corrected }}=0.025\right)$. Greater GAA1 was associated with less task-related activation in the anterior cingulate cortex $\left(t_{\max }=5.05 ; \mathrm{K}_{\mathrm{E}}=156\right.$; $\left.\mathrm{p}_{\text {corrected }}<0.001\right)$, right temporal-parietal junction $\left(t_{\max }=5.48 ; \mathrm{K}_{\mathrm{E}}=70 ; \mathrm{p}_{\text {corrected }}=0.044\right)$, and left lateral precuneus $\left(t_{\max }=5.09 ; \mathrm{K}_{\mathrm{E}}=70\right.$ $\left.\mathrm{p}_{\text {corrected }}=0.044\right)$; Figure S2.

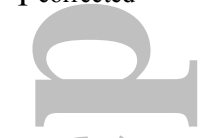

Post-hoc median-split analysis revealed several activation profiles (Figure 3). First, the bilateral dIPFC are not responsive to task demands in healthy controls, but are engaged in FRDA with no statistical differences between greater and lesser disease severity. Second, the precuneus is also not engaged in controls, but shows localized parametric increases in activation as disease severity increases. Finally, the bilateral IPS are active in controls, but this activity is increased in FRDA and a significant positive relationship with disease severity is observed in the right hemisphere.

$<<$ FIGURE $3>>$ 


\section{Network-Level Brain-Behaviour Correlations}

Based on an atlas of intrinsic functional human brain networks ${ }^{22,23}$, it was observed that all brain regions that were sensitive to FRDA disease status during single-finger tapping reside within the salience (also known as the ventral attention) network, except the superior

temporal gyrus (Figure 4A). In the FRDA cohort, greater average functional activations within ventral attention regions was associated with better performance on the offline motor precision task (Spearman's $\rho=0.40, p=0.05)$, but not speeded tapping performance $(\rho=0.16$, $\mathrm{p}=0.45)$

Similarly, all areas of statistically greater activation in the FRDA cohort during multi-finger tapping were found in the dorsal attention (Figure 4B) and executive control (Figure 4C) networks. Greater activation within regions overlapping the dorsal attention network was associated with poorer offline motor precision $(\rho=-0.65, p=0.001)$ and slower speeded tapping $(\rho=-0.41, p=0.044)$ in the FRDA cohort. Activity within the executive control network did not map significantly onto offline motor precision $(\rho=-0.31, p=0.14)$ or speeded tapping $(\rho=-0.21$, $\mathrm{p}=0.32)$.

\section{$<<$ FIGURE $4>>$}

\section{Discussion}

In individuals with FRDA, we report functional abnormalities in the cerebral cortex underlying simple self-paced, and more complex attentionally-demanding motor tasks. In the case of simple self-generated motor function, functional changes preferentially impact ventral attention (i.e., 'salience') and premotor systems. Importantly, these changes are sensitive to disease status, with increased activation relative to healthy controls evident in less severe 
FRDA, decreased activation evident in more severe disease states, and correlations between activation magnitude and motor dexterity. On the other hand, attentionally demanding motor function increased engagement of executive control and dorsal attention systems in FRDA, with activation in the latter correlated with motor performance measures. These observations are consistent with models of functional compensation operating at the level of large-scale neural networks, which may serve to mitigate disease-related dysfunction.

Models of functional compensation, although diverse in their specifics, share the premise that degeneration of neural tissues leads to a loss of functional efficiency or capacity ${ }^{17,18,24,25}$. In

response, additional neural resources may be recruited to account for neuronal damage and mitigate subsequent behavioral consequences. These compensatory mechanisms may either involve increased activation in affected task networks (i.e., neural reserve), or recruitment of alternative neural regions to complement compromised function (i.e., neural compensation $)^{24,25}$. In the current study, we observed evidence of both neural reserve and neural compensation in the cerebrum in individuals with FRDA.

Structural, functional, and connectivity abnormalities have all been previously reported in areas of the premotor and ventral attention network, including the SMA and anterior insula, in FRDA $^{4,10,26}$. However, in the current case, an inverted-U pattern of functional activation across different levels of disease severity was observed during simple finger tapping. This activation pattern is characteristic of a compensation model whereby increased neural activity is thought to mitigate functional disruptions early in the course of the disease, but which cannot be sustained with continued progression of the underlying pathology ${ }^{12,17,27}$. Similar effects have also been observed across a number other neurodegenerative disorders, including Alzheimer disease, Huntington disease, and multiple sclerosis ${ }^{12,28-31}$. This interpretation is 
further supported in the current experiment by correlations showing that individuals with greater activation in this network also have greater motor precision, providing evidence of socalled "successful compensation"12.

These non-linear cerebral functional changes occurred alongside progressive activation decreases in functionally connected regions of the cerebellum, putatively reflecting primary cerebellar pathology concurrent with secondary mechanisms of cerebral compensation.

Indeed, cerebellar changes reported in FRDA are often more strongly linked to disease expression than cerebral measures ${ }^{4,7}$. Early degeneration and dysfunction in the cerebellum may therefore be initially offset by a functional shift to relatively spared, inter-connected areas of the cerebrum. Continued cerebellar pathology, however, may eventually overwhelm the capacity limits of cerebral function, leading to dysfunction across the whole network.

The simple motor task used in this experiment was identical to that employed by Akhlaghi and colleagues (2012) in a smaller sample of 11 individuals with FRDA. In contrast to the current study, those authors reported functional deficits in cerebral premotor and supplementary motor, inferior parietal, and insula/frontal opercular regions (i.e., areas of the ventral attention network), but did not find evidence of hyperactivations. However, the clinical severity of the study sample in this earlier work lies predominantly in the more severe half of the current FARS median split. When the results from Akhlaghi et al (2012) are

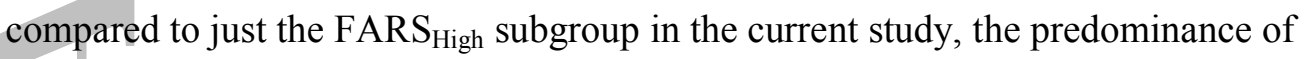
hypoactivations in these regions is replicated. Deficits in lobule V/VI and the SMA were also implicated by Ginestroni and colleagues (2011) during hand tapping. Taken together, functional changes are consistently evident in ventral attention/premotor brain regions during motor execution in individuals with FRDA. But importantly, the current study indicates that 
disease state is an important determinant of a non-linear activation profile across individuals in these regions.

During complex, attentionally demanding motor performance, neural compensation through engagement of alternative functional networks was also readily apparent. In response to task demands, individuals with FRDA activated the executive control network (bilateral dlPFC and cerebellar crus I), and activated or significantly upregulated regions of the dorsal attention network (ventrolateral premotor areas; IPS; and precuneus). Deficits in functions traditionally ascribed to these brain areas, including cognitive control and attention, have been reported in FRDA $^{8,32-34}$, but are generally considered to be subtle aspects of the disorder ${ }^{1}$. Moreover, previous functional MRI studies of cognition in FRDA have generally found a relative sparing of top-down control/attention networks ${ }^{4,8,26}$. As such, individuals with FRDA may utilize these relatively intact executive resources to compensate for impairments in other systems, perhaps by employing different cognitive strategies relative to healthy individuals to successfully mediate behavioral demands ${ }^{18,24,35}$. This shift to alternate strategies is supported by the observation of increased engagement of the dorsal attention network in individuals with FRDA who successfully completed the fMRI task, but who demonstrated worse motor precision using an offline measure. That is, individuals with greater motor impairment relied on higher-order, top-down attentional control systems to a greater degree when undertaking an attentionally-demanding behavioural task.

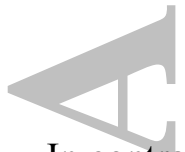

In contrast to simple motor performance, however, we do not find evidence of compensation failure in individuals with more severe disease severity during the attentionally demanding task. In fact, in subregions of the precuneus and right IPS, there is evidence of continued increases in activation with increased disease severity. These findings perhaps again provide 
evidence for the relative sparing of executive systems in FRDA, allowing neural compensation to remain effective regardless of disease status, at least within the constraints of this task. Future experiments using parametric modulations of cognitive load will be necessary to test the limits of these mechanisms ${ }^{12}$.

Neural deficits were also not apparent during attentionally demanding task performance. This finding is particularly surprising with respect to the cerebellar cortex (which was also explored at more liberal uncorrected statistical thresholds), given that deficits in lobules $\mathrm{V}$ and VI are relatively consistent in structural and functional neuroimaging studies of

FRDA $^{4,7,10,11,26}$. However, that we find cerebellar deficits in our simple motor task in the same group of participants, during the same scan session, using similar perceptual stimuli, and employing identical data processing and statistical modeling approaches provides some protection against type-II error. While definitive explanations for this intact cerebellar activation are beyond the scope of the current data, there are several compelling possibilities. First, it may be that the functional reorganization of neural processing from impaired ventral attention systems (including lobule VI) to intact executive systems prevents the former from becoming overloaded ${ }^{35}$. Alternatively, inputs down the rostral-caudal prefrontal hierarchy from higher-order executive to lower-order lateral premotor areas ${ }^{36}$, and subsequently through premotor and motor cortico-cerebellar loops ${ }^{37}$, may sustain task-appropriate neuronal activity. These hypotheses are speculative, but present interesting avenues for future research.

Notably, the simple and complex tasks were selectively sensitive to dysfunction in bottom-up (i.e., salience) and top-down (i.e., executive/dorsal attention) brain networks, respectively. This selectivity may relate to the subjective self-assessment and internal vigilance demands of the self-paced manipulation, relative to the objective action-outcome evaluation and external 
vigilance requirements of the visually-cued task. The current study thus supports dysfunction in both processes in FRDA, the consequences of which may manifest independently in different contexts.

Definitive inference of links between cerebral compensation, disease severity, and disease progression are limited by the cross-sectional design of this work. Future longitudinal studies will be necessary to confirm these relationships. Furthermore, due to the technological limitations of fMRI and the behavioral limitations inherent to individuals with FRDA, it was necessary to restrict experimentation of motor function to hand movements (as opposed to, for example, gait impairments or complex real-world behaviours). While the ecological validity of such designs may be questionable, the ability to isolate fundamental dysfunctions is useful in developing more complete neurobiological models of this disorder. Furthermore, while links between brain function and disease severity are reported in this work, investigating the potential modulatory role of differences in age-at-onset ${ }^{34}$ was not possible due to confounds with normal aging processes in this sample (age-at-onset vs. current age: $r=0.84$ ); further investigations decoupling these effects are warranted.

Contemporary theories, models, and evidence of neural compensation and neural reserve firmly support the notion that neurofunctional changes underlying degenerative processes are not only detrimental, but may also be compensatory ${ }^{12,25,28}$. This idea has driven a groundswell of research into novel therapeutic approaches designed to stimulate or maintain these compensatory cerebral processes through, e.g., non-invasive brain stimulation ${ }^{38,39}$ or cognitive therapies ${ }^{40,41}$. Given the evidence that cerebral compensation may also operate in FRDA, these novel means of intervention represent exciting pathways for future research. 
In summary, the present study motivates a reconceptualization of cerebral contributions to FRDA. In particular, cerebral alterations are likely not merely the secondary detriments of cerebellar and spinal insults. Rather, cerebral changes may represent capacity limited compensatory processes operating at the network level of brain function, which serve to mitigate the behavioral consequences of progressive disease-related pathology. Critically, these novel findings inform development of new avenues of clinical intervention for FRDA.

(1)

\section{Acknowledgements}

This study was supported by funding from the Australian National Health and Medical Research Council (Project Grant 1046037; Fellowship 1106533 to IHH). We thank the individuals with Friedreich ataxia and healthy controls who participated in this study. The authors declare no conflicts of interest.

\section{Author Roles}

IHH conducted the statistical analyses and wrote the paper; LAC and MBD conceptualized the project, coordinated participant recruitment, and edited the paper; GFE and NG-K conceptualized the project, consulted on the analysis approach, and edited the paper; ES conceptualized the project and edited the paper; MRS recruited the participants, collected/managed the data, conducted statistical analyses, and edited the paper. 


\section{References}

1. Pandolfo M. Friedreich ataxia. Archives of neurology 2008;65(10):1296-1303.

2. Akhlaghi H, Yu J, Corben L, et al. Cognitive deficits in Friedreich ataxia correlate with micro-structural changes in dentatorubral tract. Cerebellum (London, England) 2014;13(2):187-198.

3. Zalesky A, Akhlaghi H, Corben LA, et al. Cerebello-cerebral connectivity deficits in Friedreich ataxia. Brain structure \& function 2014;219(3):969-981.

4. Harding IH, Corben LA, Storey E, et al. Fronto-cerebellar dysfunction and dysconnectivity underlying cognition in friedreich ataxia: The IMAGE-FRDA study. Human brain mapping 2016;37(1):338-350.

5. Selvadurai LP, Harding IH, Corben LA, et al. Cerebral and cerebellar grey matter atrophy in Friedreich ataxia: the IMAGE-FRDA study. Journal of neurology 2016.

6. Harding IH, Raniga P, Delatycki MB, et al. Tissue atrophy and elevated iron concentration in the extrapyramidal motor system in Friedreich ataxia: the IMAGE-FRDA study. Journal of neurology, neurosurgery, and psychiatry 2016.

7. Rezende TJ, Silva CB, Yassuda CL, et al. Longitudinal magnetic resonance imaging study shows progressive pyramidal and callosal damage in Friedreich's ataxia. Movement disorders : official journal of the Movement Disorder Society 2016;31(1):70-78.

8. Dogan I, Tinnemann E, Romanzetti S, et al. Cognition in Friedreich's ataxia: a behavioral and multimodal imaging study. Annals of clinical and translational neurology 2016;3(8):572-587.

9. Corben LA, Georgiou-Karistianis N, Bradshaw JL, Evans-Galea MV, Churchyard AJ, Delatycki MB. Characterising the neuropathology and neurobehavioural phenotype in Friedreich ataxia: a systematic review. Advances in experimental medicine and biology 2012;769:169-184. 
10. Akhlaghi H, Corben L, Georgiou-Karistianis N, et al. A functional MRI study of motor dysfunction in Friedreich's ataxia. Brain research 2012;1471:138-154.

11. Ginestroni A, Diciotti S, Cecchi P, et al. Neurodegeneration in friedreich's ataxia is associated with a mixed activation pattern of the brain. A fMRI study. Human brain mapping 2012;33(8):1780-1791.

12. Scheller E, Minkova L, Leitner M, Kloppel S. Attempted and successful compensation in preclinical and early manifest neurodegeneration - a review of task FMRI studies. Frontiers in psychiatry 2014;5:132.

13. Carrera E, Tononi G. Diaschisis: past, present, future. Brain : a journal of neurology 2014;137(Pt 9):2408-2422.

14. Corben LA, Delatycki MB, Bradshaw JL, et al. Impairment in motor reprogramming in Friedreich ataxia reflecting possible cerebellar dysfunction. Journal of neurology 2010;257(5):782-791.

15. Corben LA, Georgiou-Karistianis N, Bradshaw JL, Hocking DR, Churchyard AJ, Delatycki MB. The Fitts task reveals impairments in planning and online control of movement in Friedreich ataxia: reduced cerebellar-cortico connectivity? Neuroscience $2011 ; 192: 382-390$

16. Ridderinkhof KR, van den Wildenberg WP, Segalowitz SJ, Carter CS. Neurocognitive mechanisms of cognitive control: the role of prefrontal cortex in action selection, response inhibition, performance monitoring, and reward-based learning. Brain and cognition 2004;56(2):129-140.

17. Reuter-Lorenz PA, Cappell KA. Neurocognitive aging and the compensation hypothesis. Curr Dir Psychol Sci 2008;17(3):177-182.

18. Park DC, Reuter-Lorenz P. The adaptive brain: aging and neurocognitive scaffolding. Annual review of psychology 2009;60:173-196. 
19. Stout JC, Paulsen JS, Queller S, et al. Neurocognitive signs in prodromal Huntington disease. Neuropsychology 2011;25(1):1-14.

20. Behzadi Y, Restom K, Liau J, Liu TT. A component based noise correction method (CompCor) for BOLD and perfusion based fMRI. NeuroImage 2007;37(1):90-101.

21. Subramony SH, May W, Lynch D, et al. Measuring Friedreich ataxia: Interrater reliability of a neurologic rating scale. Neurology 2005;64(7):1261-1262.

22. Buckner RL, Krienen FM, Castellanos A, Diaz JC, Yeo BT. The organization of the human cerebellum estimated by intrinsic functional connectivity. Journal of neurophysiology $2011 ; 106(5): 2322-2345$.

23. Yeo BT, Krienen FM, Sepulcre J, et al. The organization of the human cerebral cortex estimated by intrinsic functional connectivity. Journal of neurophysiology 2011;106(3):1125-

\section{5}

24. Barulli D, Stern Y. Efficiency, capacity, compensation, maintenance, plasticity: emerging concepts in cognitive reserve. Trends in cognitive sciences 2013;17(10):502-509. 25

25. Fornito A, Zalesky A, Breakspear M. The connectomics of brain disorders. Nature reviews Neuroscience 2015;16(3):159-172.

26. Georgiou-Karistianis N, Akhlaghi H, Corben LA, et al. Decreased functional brain activation in Friedreich ataxia using the Simon effect task. Brain and cognition 2012;79(3):200-208.

27. Saxena S, Caroni P. Selective neuronal vulnerability in neurodegenerative diseases: from stressor thresholds to degeneration. Neuron 2011;71(1):35-48.

28. Schoonheim MM, Filippi M. Functional plasticity in MS: friend or foe? Neurology 2012;79(14):1418-1419. 
29. Georgiou-Karistianis N, Poudel GR, Dominguez DJ, et al. Functional and connectivity changes during working memory in Huntington's disease: 18 month longitudinal data from the IMAGE-HD study. Brain and cognition 2013;83(1):80-91.

30. Poudel GR, Stout JC, Dominguez DJ, et al. Functional changes during working memory in Huntington's disease: 30-month longitudinal data from the IMAGE-HD study. Brain structure \& function 2015;220(1):501-512.

31. Kloppel S, Draganski B, Siebner HR, Tabrizi SJ, Weiller C, Frackowiak RS.

Functional compensation of motor function in pre-symptomatic Huntington's disease. Brain : a journal of neurology 2009;132(Pt 6):1624-1632.

32. Corben LA, Akhlaghi H, Georgiou-Karistianis N, et al. Impaired inhibition of prepotent motor tendencies in Friedreich ataxia demonstrated by the Simon interference task. Brain and cognition 2011;76(1):140-145.

33. Klopper F, Delatycki MB, Corben LA, Bradshaw JL, Rance G, Georgiou-Karistianis

$\mathrm{N}$. The test of everyday attention reveals significant sustained volitional attention and working memory deficits in friedreich ataxia. Journal of the International Neuropsychological Society: JINS 2011;17(1):196-200.

34. Nachbauer W, Bodner T, Boesch S, et al. Friedreich ataxia: executive control is related to disease onset and GAA repeat length. Cerebellum (London, England) 2014;13(1):9-

16.

35. Noppeney U, Friston KJ, Price CJ. Degenerate neuronal systems sustaining cognitive functions. Journal of anatomy 2004;205(6):433-442.

36. Badre D. Cognitive control, hierarchy, and the rostro-caudal organization of the frontal lobes. Trends in cognitive sciences 2008;12(5):193-200. 
37. Kelly RM, Strick PL. Cerebellar loops with motor cortex and prefrontal cortex of a nonhuman primate. The Journal of neuroscience : the official journal of the Society for Neuroscience 2003;23(23):8432-8444.

38. Miniussi C, Cappa SF, Cohen LG, et al. Efficacy of repetitive transcranial magnetic stimulation/transcranial direct current stimulation in cognitive neurorehabilitation. Brain stimulation 2008;1(4):326-336.

39. Floel A. tDCS-enhanced motor and cognitive function in neurological diseases.

NeuroImage 2014;85 Pt 3:934-947.

40. Valenzuela M, Sachdev P. Can cognitive exercise prevent the onset of dementia?

Systematic review of randomized clinical trials with longitudinal follow-up. The American journal of geriatric psychiatry : official journal of the American Association for Geriatric Psychiatry 2009;17(3):179-187.

41. Simon SS, Yokomizo JE, Bottino CM. Cognitive intervention in amnestic Mild Cognitive Impairment: a systematic review. Neuroscience and biobehavioral reviews 2012;36(4):1163-1178.

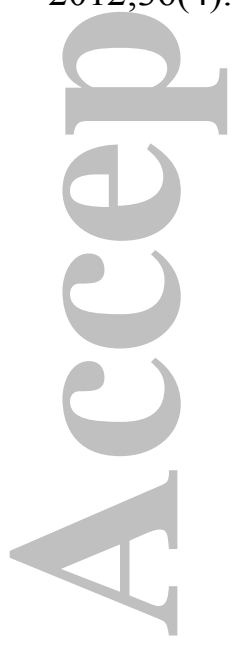




\section{Figure Legends}

Figure 1: Within-Group Task fMRI Activations.

Functional activations during Self-Paced Single Finger (Top) and Cued Multi-Finger (Bottom) Tasks (FWE-corrected $p_{\text {cluster }}<0.05$ with cluster-forming $p_{\text {voxel }}<0.001$ ). Activations specific to controls are displayed in red, to FRDA in green, and common to both in yellow.

Figure 2: Between-Group fMRI Activations during Self-Paced Tapping.

Significant group functional differences (FRDA vs. Controls) in the cerebellum (left) and clinical correlations in the cerebrum (right) during self-paced single finger tapping. Bar graphs illustrate mean group fMRI activations $( \pm \mathrm{SD})$, relative to resting baseline, for controls (blue), and a median split of the FRDA group into low disease severity (red), and high disease severity,(green) subgroups. FARS, Friedreich Ataxia Rating Scale; STG, superior temporal gyus; IPL, inferior parietal lobule; SMA, supplementary motor area.

Figure 3: Between-Group fMRI Activations during Cued Multi-Finger Tapping. Significant group functional differences (FRDA vs. Controls) in the cerebrum during cued multi-finger tapping. Bar graphs illustrate mean group fMRI activations $( \pm \mathrm{SD})$, relative to resting baseline, for controls (blue), and a median split of the FRDA group into low disease severity (red), and high disease severity (green) subgroups. FARS, Friedreich Ataxia Rating Scale; IPS, intraparietal sulcus; dlPFC, dorsolateral prefrontal cortex.

Figure 4: Network-level Correlations with Offline Motor Behaviour Performance. Linear correlations between offline motor precision and fMRI activation magnitude during self-paced single finger tapping (SP) in disease-relevant brain areas (orange-red; see Figure 2) that overlap with the ventral attention brain network (A; cyan), and fMRI activation magnitude during externally-cued multi-finger tapping (MF) and disease-relevant brain areas (orange-red; see Figure 3) that overlap with the dorsal attention (B; blue) and executive control (C; green) brain networks. Brain network illustration modified from Yeo et al, 2011. Spearman correlation coefficients are reported $(* p<0.05, * * p<0.001)$.

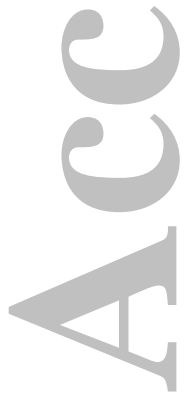


Table 1. Demographic, Clinical, and Task Performance Characteristics

\begin{tabular}{lllll}
\hline & FRDA $(\boldsymbol{N}=\mathbf{2 5})$ & Controls $(\boldsymbol{N}=\mathbf{3 3})$ & Statistic & p-value \\
\hline Demographics & & & & \\
\hline Age, years & $34.9 \pm 12.1$ & $36.9 \pm 13.1$ & $t(56)=0.60$ & $p=0.55$ \\
Gender (M, F) & 15,10 & 16,17 & $\chi^{2}(1)=0.76$ & $p=0.38$ \\
\hline Clinical data & & & - \\
\hline Disease Severity, FARS & $77.4 \pm 23.7$ & - & - & - \\
Disease Duration, years & $14.9 \pm 6.6$ & - & - & - \\
Age of onset, years & $19.8 \pm 9.3$ & - & - & - \\
GAA1 repeat length & $553 \pm 232$ & - & - & - \\
GAA2 repeat length & $844 \pm 221$ & - & - & $\boldsymbol{p}=\mathbf{0 . 0 2 2}$ \\
\hline Online Motor Task Performance & & & $p=0.90$ \\
\hline Self-paced, Hz & $0.80 \pm 0.18$ & $0.70 \pm 0.16$ & $t(56)=2.35$ & \\
Multi-finger, \% Accuracy & $100[89-100]$ & $98[92-100]$ & $U=405 ; Z=0.13$ & \\
\hline Offline Motor Task Performance & & & $\boldsymbol{p}<\mathbf{0 . 0 0 1}$ \\
\hline Speeded Tapping, Hz & $2.40 \pm 0.65$ & $4.91 \pm 0.54$ & $t(55)=15.9$ & $\boldsymbol{p}<\mathbf{0 . 0 0 1}$ \\
\hline Paced Tapping, Precision & $12.38 \pm 4.47$ & $22.34 \pm 5.79$ & $t(55)=7.04$ & - \\
\hline
\end{tabular}

GAA1, smaller $F X N$ intron 1 GAA repeat size; GAA2, larger $F X N$ intron 1 GAA repeat size; $\boldsymbol{F A R S}$, Friedreich Ataxia Räting Scale; Values are Mean \pm S.D. unless otherwise indicated; ${ }^{a}$ Median[Range] is reported due to strong ceiling effects

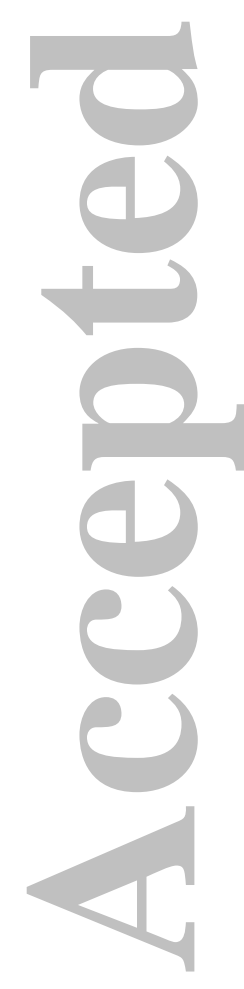



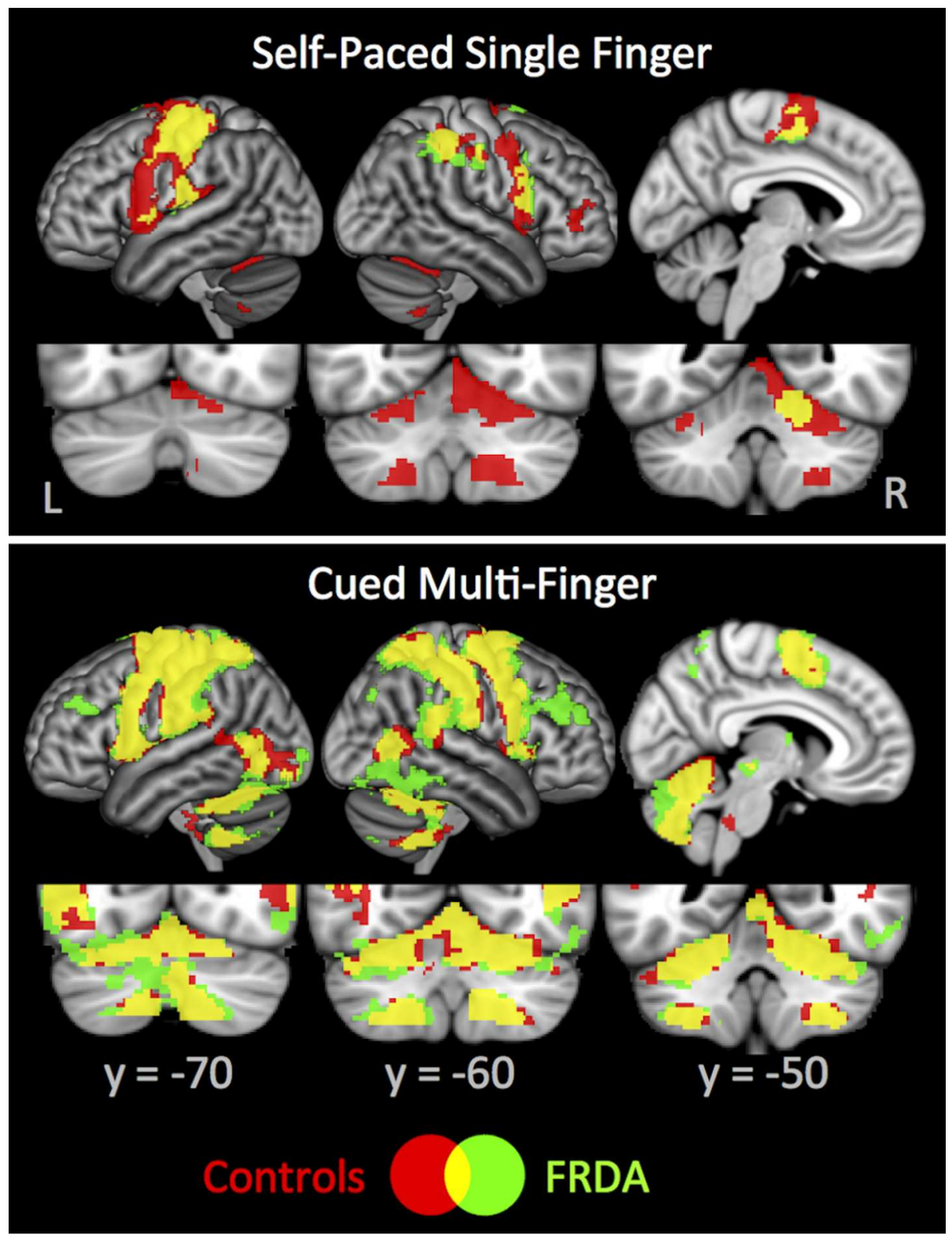

Within-Group Task fMRI Activations. Functional activations during Self-Paced Single Finger (Top) and Cued Multi-Finger (Bottom) Tasks (FWE-corrected pcluster $<0.05$ with cluster-forming pvoxel<0.001). Activations specific to controls are displayed in red, to FRDA in green, and common to both in yellow.

Figure 1

$85 \times 111 \mathrm{~mm}(300 \times 300$ DPI $)$ 

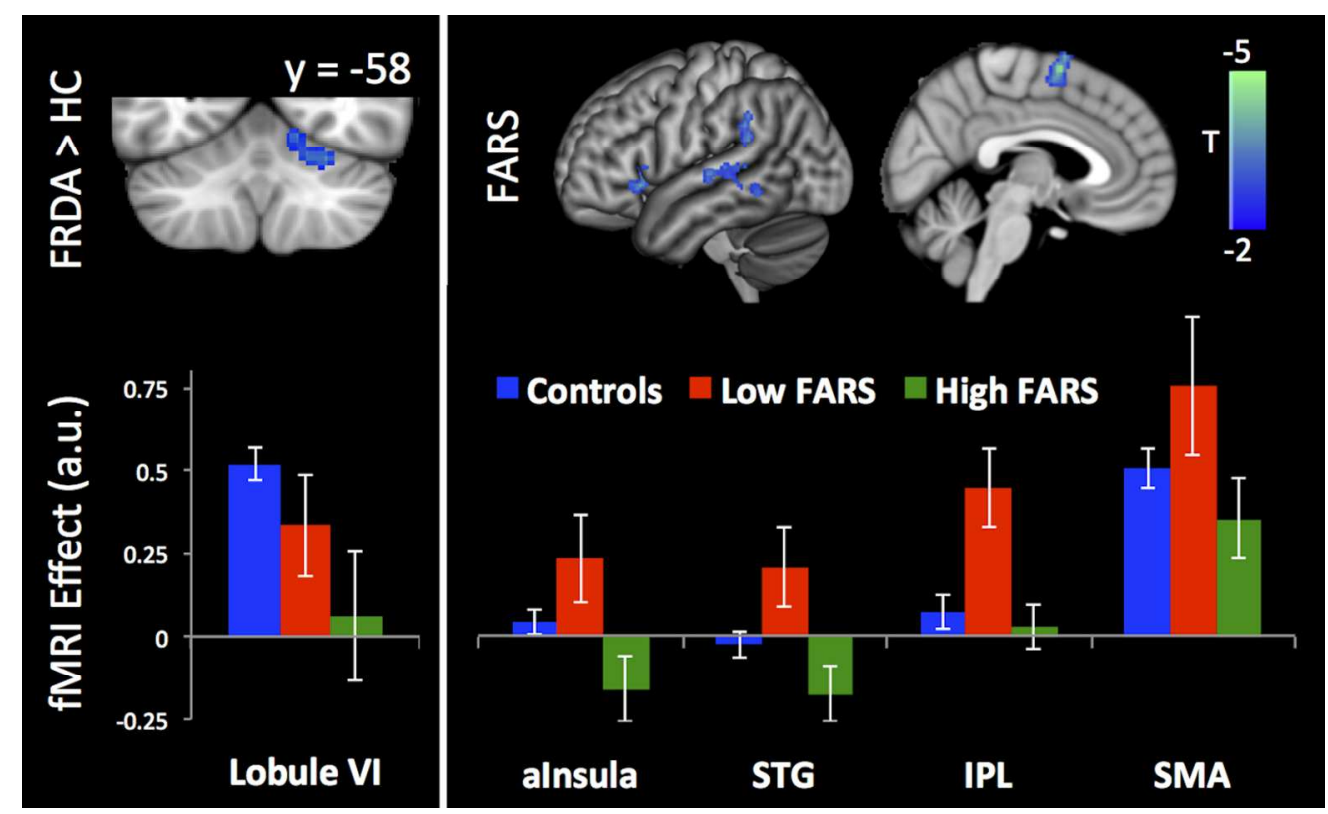

Between-Group fMRI Activations during Self-Paced Tapping. Significant group functional differences (FRDA vs. Controls) in the cerebellum (left) and clinical correlations in the cerebrum (right) during self-paced single finger tapping. Bar graphs illustrate mean group fMRI activations ( \pm SD), relative to resting baseline, for controls (blue), and a median split of the FRDA group into low disease severity (red), and high disease severity (green) subgroups. FARS, Friedreich Ataxia Rating Scale; STG, superior temporal gyus; IPL, inferior parietal lobule; SMA, supplementary motor area.

Figure 2

$119 \times 73 \mathrm{~mm}(300 \times 300$ DPI $)$

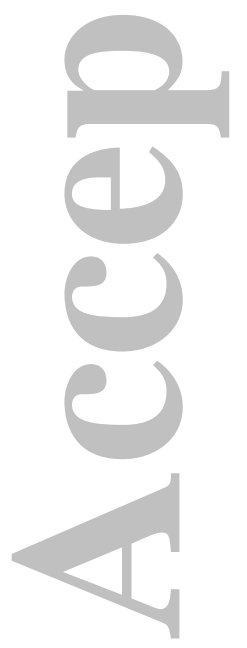




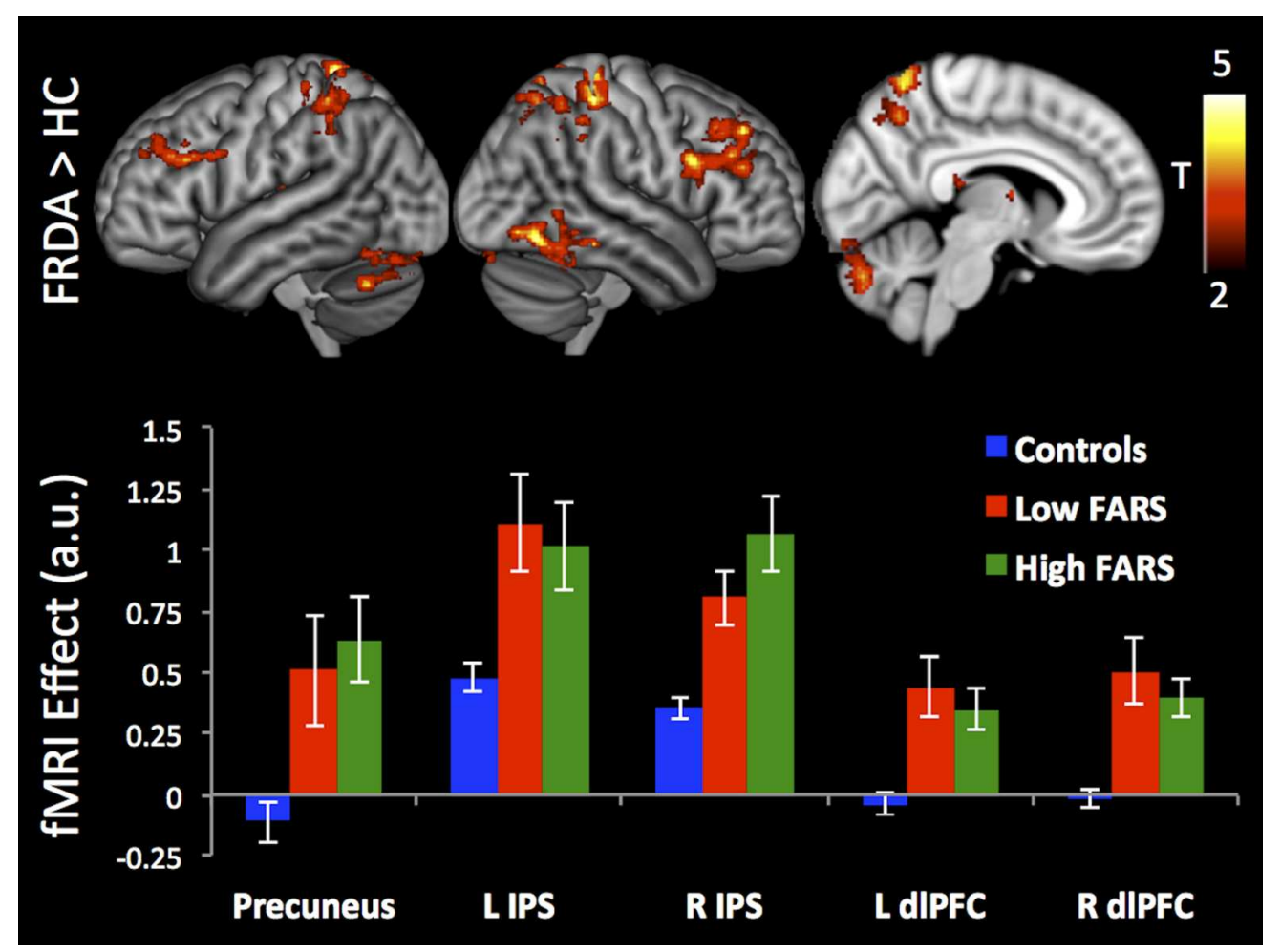

Between-Group fMRI Activations during Cued Multi-Finger Tapping. Significant group functional differences (FRDA vs. Controls) in the cerebrum during cued multi-finger tapping. Bar graphs illustrate mean group fMRI activations ( $\pm S D$ ), relative to resting baseline, for controls (blue), and a median split of the FRDA group into low disease severity (red), and high disease severity (green) subgroups. FARS, Friedreich Ataxia Rating Scale; IPS, intraparietal sulcus; dIPFC, dorsolateral prefrontal cortex.

Figure 3

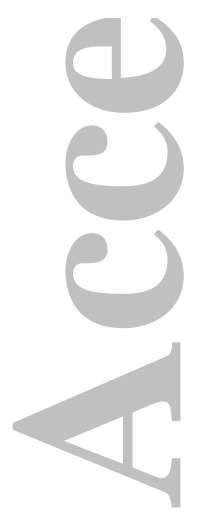

$119 \times 89 \mathrm{~mm}(300 \times 300$ DPI $)$ 


\section{Salience Network}
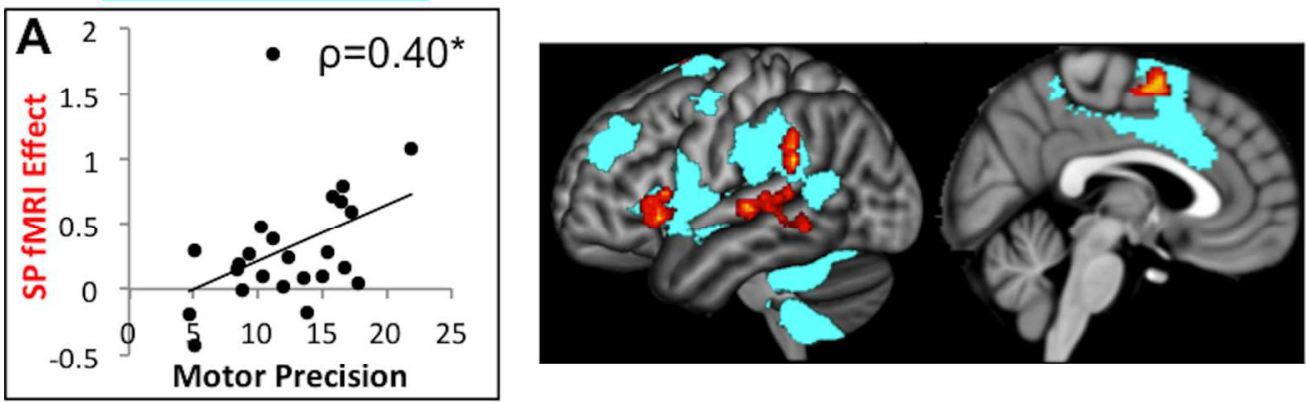

\section{Dorsal Attention Network Executive Control Network}
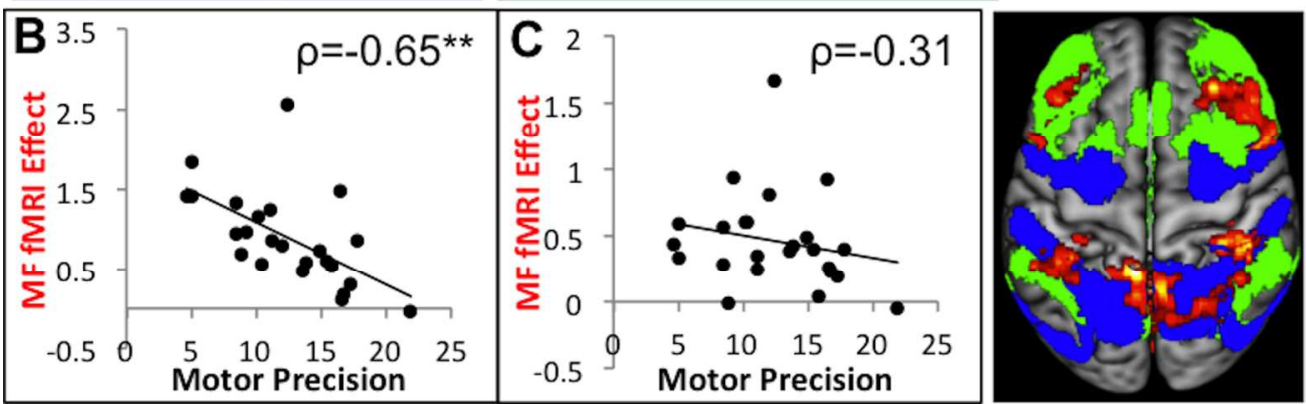

Network-level Correlations with Offline Motor Behaviour Performance. Linear correlations between offline motor precision and fMRI activation magnitude during self-paced single finger tapping (SP) in diseaserelevant brain areas (orange-red; see Figure 2 ) that overlap with the ventral attention brain network ( $A$; cyan), and fMRI activation magnitude during externally-cued multi-finger tapping (MF) and disease-relevant brain areas (orange-red; see Figure 3 ) that overlap with the dorsal attention (B; blue) and executive control (C; green) brain networks. Brain network illustration modified from Yeo et al, 2011. Spearman correlation coefficients are reported $(* p<0.05, * * p<0.001)$.

Figure 4

$129 \times 94 \mathrm{~mm}(300 \times 300$ DPI $)$

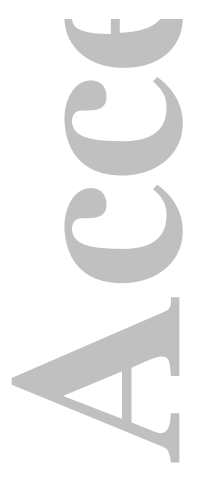




\section{Cerebral Compensation during Motor Function in Friedreich Ataxia: The IMAGE- FRDA Study}

\section{SUPPLEMENTARY MATERIALS}

Ian H. Harding ${ }^{1}$, Louise A. Corben ${ }^{1,2}$, Martin B. Delatycki ${ }^{1,2,3}$, Monique R. Stagnitti ${ }^{1}$, Elsdon Storey $^{4}$, Gary F. Egan ${ }^{1,5}$, Nellie Georgiou-Karistianis ${ }^{1}$

School of Psychological Sciences \& Monash Institute of Cognitive and Clinical Neuroscience, Monash University, Melbourne, Australia ${ }^{2}$ Bruce Lefroy Centre, Murdoch Childrens Research Institute, Melbourne, Australia ${ }^{3}$ Clinical Genetics, Austin Health, Melbourne, Australia ${ }^{4}$ Department of Medicine, Monash University, Melbourne, Australia ${ }^{5}$ Monash Biomedical Imaging, Monash University, Melbourne, Australia

Correspondence:

Dr. Ian Harding

School of Psychological Sciences

18 Innovation Walk

Monash University, VIC, 3800

Australia

Email: ian.harding@monash.edu

Phone:+61-3-9905-9283

Fax: +61-3-9905-3948 


\section{The IMAGE-FRDA Study}

The IMAGE-FRDA study is a Melbourne-based longitudinal investigation of the neural and cognitive correlates of Friedreich ataxia. Participants partake in two identical research sessions separated by 2 years, which include a behavioural assessment and magnetic resonance imaging (MRI) scan. The study has enrolled 33 individuals with FRDA and 37 healthy controls at the first timepoint. At the time of writing, data collection for the second timepoint is underway.

Each data collection session begins with collection of written informed consent, followed by a neuropsychological battery (45-60mins), finger-tapping measures of motor ability (8-10mins), and preparation for the MRI scan ( $\sim 15 \mathrm{mins})$, including safety screening and introduction to the tasks that will be performed during scanning. Participants then undergo a 1-hour MRI sean, which includes measures of brain anatomy, connectivity, chemical composition, and function associated with motor and cognitive tasks.

The current paper focuses principally on the motor measures recorded during MRI scanning. Relationships with the motor measures acquired during the behavioural assessment are also assessed. Descriptions and depictions of each of these motor tasks are provided in

Supplementary Figure 1, below, and in the main text of the manuscript.

\section{Participant Exclusions}

Data from 8 individuals with FRDA were excluded from analysis:

- 2 did not tap during the self-paced tapping task

- 1 performed very poorly on the cued tapping task (5.4 s.d. below the mean)

- 1 fell asleep during a portion of the task period

- 2 were excluded due to excessive motion ( $>20 \%$ of volumes with $>0.5 \mathrm{~mm}$ motion)

- 2 fMRI datasets were corrupted and not usable

Data from 4 healthy individuals were excluded from analysis:

- 1 tapped excessively fast during the self-paced tapping task (3.9 s.d. above the mean)

- 1 fell asleep during a portion of the task period

- 1 did not have complete data coverage of the cerebellum

- 1 did not have behavioural recordings during scanning due to a technical issue 


\section{Self-Paced Functional Group Differences in a Behavioural-Matched Subgroup}

On average, the FRDA cohort tapping significantly faster than the control cohort during the online self-paced tapping task (see Table 1). Tapping rate was included in group statistical models to regress out performance-related group differences in the underlying brain activations. However, in order to provide an additional check that behavioural differences were not driving the observed activation difference in lobule VI (Figure 2; Table S2), a secondary analysis using a behaviourally-matched subgroup was undertaken.

After excluding the 8 slowest tapping control participants, the remaining 25 controls (mean $=0.76 \mathrm{~Hz}, \mathrm{SD}=0.13 \mathrm{~Hz}$ ) were behaviourally-matched with the 25 individuals with FRDA (mean $\left.=0.80 \mathrm{~Hz}, \mathrm{SD}=0.18 \mathrm{~Hz} ; \mathrm{t}_{48}=0.97, \mathrm{p}=0.34\right)$. Age $(\mathrm{p}=0.38)$ and gender $(\mathrm{p}=0.57)$ remained matched, and were retained in regression models.

Region-of-interest regressions and t-tests on the functional activations in lobule VI revealed comparable between-group effect sizes between the full cohort $\left(r_{\text {partial }}=0.41\right.$, Cohen's $\left.d=0.67\right)$ and the subsample ( $\mathrm{r}_{\text {partial }}=0.38$, Cohen's $\mathrm{d}=0.62$ ).

This result supports the presence of FRDA-related functional abnormalities in cerebellar lobule VI, independent of behavioural task performance.

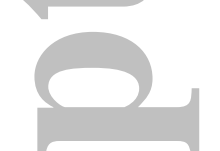

\section{Relationships between Brain Atrophy and Brain Function}

Within brain regions showing significant functional differences in the FRDA cohort, there

was also significantly less grey matter volume in cerebellar lobule VI $\left(\mathrm{t}_{56}=2.94, \mathrm{p}=0.005\right)$ and crus I $\left(\mathrm{t}_{56}=2.30, \mathrm{p}=0.025\right.$; all others, $\left.\mathrm{p}>0.13\right)$. However, structure-function correlations were not statistically significant (lobule VI: $\mathrm{r}_{58}=0.22, \mathrm{p}=0.095$; crus $\mathrm{I}: \mathrm{r}_{58}=-0.21, \mathrm{p}=0.12$ ).

Therefore, although functional abnormalities were co-located with structural changes in the cerebellum, there was not convincing evidence that structural changes significantly accounted for any of this functional variance (within the bounds of the available statistical power). Cerebral functional changes appeared to occur entirely independent of structural atrophy. 


\section{A. Speeded, Offline Tapping}

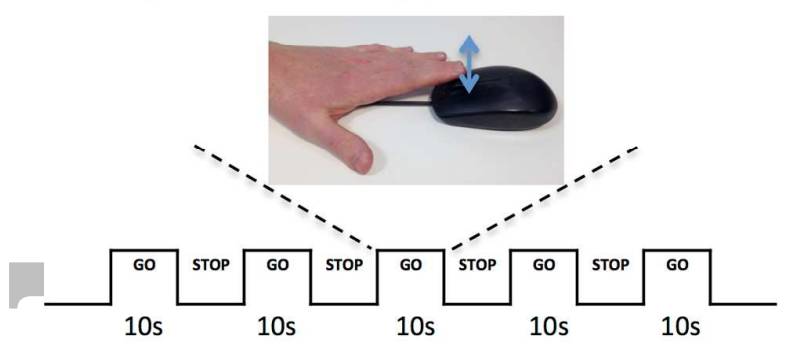

Click mouse button as quickly as possible with the nondominant index finger. To avoid fatigue effects, rest time between blocks is determined by the participant.

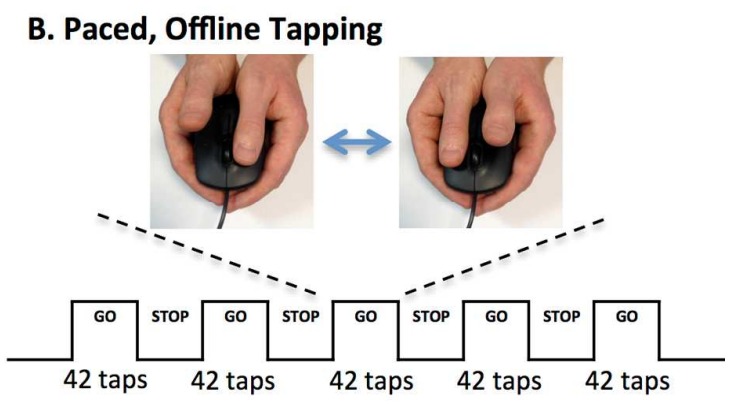

Participants first match a $1.8 \mathrm{~Hz}$ tone for 11 taps, then continue for 31 taps ( 24 secs total) after tone has stopped, alternating thumbs with each tap. Rest time between blocks is determined by the participant.

\section{Self-Paced, Online Tapping Task (Single Finger)}

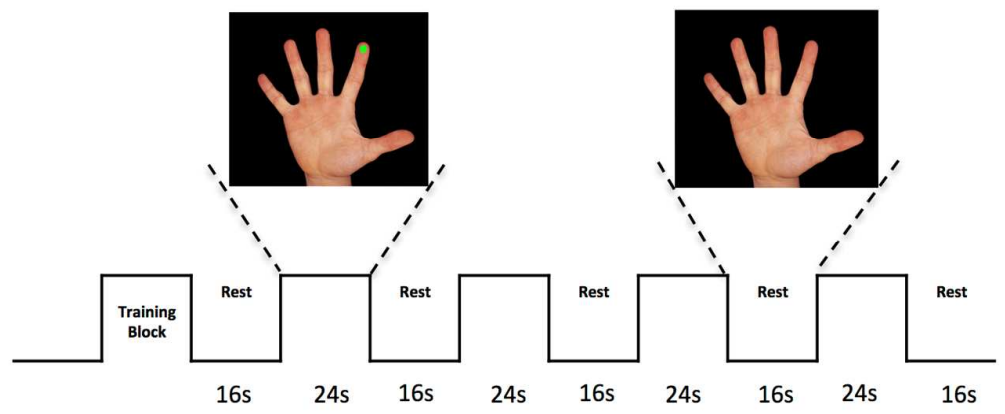

Participants are trained to tap the thumb to the index finger at $0.67 \mathrm{~Hz}$ during the training block using a visual cue (flashing green dot). During task blocks, participants aim to tap at the trained rate when the cue (solid green dot) appears.

\section{Visually-Paced, Online Tapping (Multiple Fingers)}

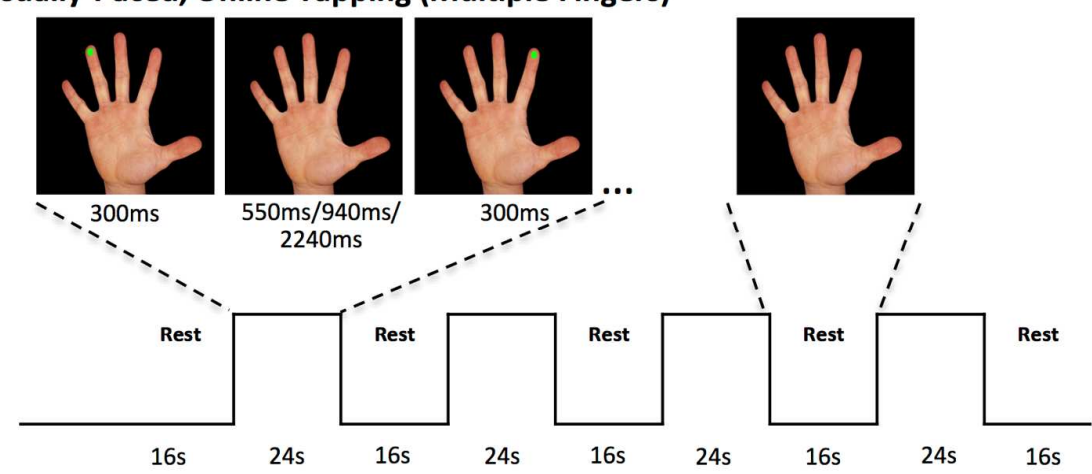

Participants are visually cued (green dot) to tap the index, middle, ring, or little finger to the thumb in a pseudo-randomised order. Each stimulus is displayed for $300 \mathrm{~ms}$, with a varying intertap-interval averaging $1200 \mathrm{~ms}$ ( $550 \mathrm{~ms}, 940 \mathrm{~ms}$, or $2240 \mathrm{~ms}$ ). Each block consists of 16 taps ( 4 of each finger).

Figure S1: Experimental Design. The two offline tasks (A and B) are undertaken prior to the scanning session to provide measures of maximal speeded digit movement (A) and motor precision (B). The two online tasks (C and D), counter-balanced in order across participants, are performed during functional MRI recordings to assess simple motor generation (C) and more complex and attentionally-demanding motor generation (D). 


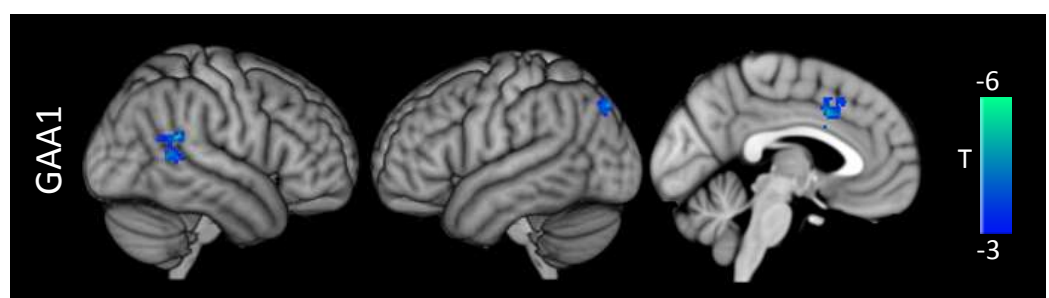

Figure S2: Significant negative correlations between GAA1 and multi-finger task activations, controlling for FARS score. Whole-brain FWE-corrected $\mathrm{p}<0.05$ (cluster-forming threshold, $\left.\mathrm{p}<0.001, \mathrm{~K}_{\mathrm{E}}>70\right)$. 
Table S1: Within-Group Functional Activations During Self-Paced and Cued Motor Tasks

\begin{tabular}{|c|c|c|c|c|c|c|c|c|c|c|c|}
\hline \multirow[b]{2}{*}{ Region (BA) } & & \multicolumn{5}{|c|}{ FRDA } & \multicolumn{5}{|c|}{ CONTROLS } \\
\hline & & $K_{E}$ & $x$ & $y$ & $z$ & $t_{\max }$ & $K_{E}$ & $x$ & $y$ & $z$ & $t_{\max }$ \\
\hline Self-Paced, Single Finger & & & & & & & & & & & \\
\hline Primary Motor Cortex (4) & $\mathrm{L}$ & 363 & -38 & -20 & 50 & 7.97 & 1282 & -36 & -20 & 50 & 13.3 \\
\hline Primary Somatosensory Cortex (3) & $\mathrm{L}$ & & -48 & -18 & 48 & 7.80 & & -48 & -18 & 48 & 10.5 \\
\hline Supplementary Motor Area (6) & - & 31 & -4 & -8 & 50 & 7.45 & 299 & 0 & 0 & 58 & 9.44 \\
\hline Inferior Parietal Lobule (40) & $\mathrm{R}$ & 12 & 62 & -34 & 48 & 7.99 & 1 & 60 & -34 & 48 & 5.68 \\
\hline Cerebellum, Lobule V-VI & $\mathrm{R}$ & 3 & 14 & -52 & -20 & 5.94 & 531 & 16 & -50 & -22 & 11.5 \\
\hline Ventral Premotor Cortex (6/44) & $\mathrm{L}$ & & & & & & 11 & -58 & 6 & 26 & 6.16 \\
\hline Ventral Premotor Cortex (6/44) & $\mathrm{R}$ & & & & & & 6 & 60 & 12 & 24 & 6.09 \\
\hline Posterior Insula (13) & $\mathrm{L}$ & & & & & & 43 & -48 & -24 & 18 & 7.64 \\
\hline Middle Insula (13) & $\mathrm{L}$ & & & & & & 61 & -46 & 0 & 4 & 7.13 \\
\hline $\begin{array}{l}\text { Cerebellum, Lobule VIIIb } \\
\text { Cued/Multi-Finger }\end{array}$ & $\mathrm{R}$ & & & & & & 60 & 18 & -60 & -50 & 7.26 \\
\hline Primary Motor Cortex (4) & $\mathrm{L}$ & 2725 & -36 & -22 & 52 & 9.5 & 4642 & -38 & -22 & 52 & 16.9 \\
\hline Primary Somatosensory Cortex (1-3) & $\mathrm{L}$ & & -48 & -22 & 48 & 11.0 & & -48 & -22 & 48 & 14.5 \\
\hline Inerior Parietal Lobule (40) & $\mathrm{L}$ & & -48 & -30 & 50 & 11.4 & & -46 & -30 & 50 & 15.5 \\
\hline Dorsal Premotor Cortex (6) & $\mathrm{L}$ & & -26 & -14 & 58 & 8.0 & & -24 & -6 & 54 & 11.1 \\
\hline Ventral Premotor Cortex $(9 / 6)$ & $\mathrm{L}$ & 109 & -54 & 2 & 36 & 9.1 & & -54 & 2 & 36 & 13.0 \\
\hline Superior Parietal Lobule (7) & $\mathrm{L}$ & 55 & -20 & -62 & 56 & 7.0 & & -18 & -64 & 58 & 8.3 \\
\hline Inferior Parietal Lobule (40) & $\mathrm{R}$ & 1010 & 46 & -36 & 56 & 10.9 & 1424 & 46 & -36 & 54 & 8.5 \\
\hline Primary Somatosensory Cortex (1-3) & $\mathrm{R}$ & & 54 & -20 & 44 & 8.7 & & 52 & -24 & 44 & 9.2 \\
\hline Superior Parietal Lobule (7) & $\mathrm{R}$ & 71 & 26 & -54 & 60 & 7.7 & & 26 & -52 & 60 & 9.1 \\
\hline Dorsal Premotor Cortex (6) & $\mathrm{R}$ & 499 & 38 & -4 & 64 & 9.6 & 858 & 38 & -2 & 62 & 10.9 \\
\hline Ventral Premotor Cortex $(9 / 6)$ & $\mathrm{R}$ & 110 & 60 & 12 & 24 & 7.7 & 372 & 60 & 10 & 26 & 8.5 \\
\hline Supplementary Motor Area (6) & - & 338 & -4 & 6 & 52 & 8.3 & 846 & -2 & 2 & 54 & 12.0 \\
\hline Middle Insula (13) & $\mathrm{L}$ & 41 & -40 & -4 & 14 & 8.5 & 88 & -40 & -4 & 12 & 9.4 \\
\hline Cerebellum, Lobule V-VI & $\mathrm{R}$ & 488 & 14 & -52 & -24 & 10.7 & 1840 & 16 & -52 & -20 & 16.4 \\
\hline Cerebellum: Lobule VIIIa/b & $\mathrm{R}$ & 399 & 12 & -62 & -46 & 9.1 & & 12 & -62 & -46 & 11.9 \\
\hline Cerebellum: Lobule VIIb & $\mathrm{R}$ & & 10 & -74 & -50 & 8.4 & & 6 & -72 & -44 & 8.6 \\
\hline Cerebellum: Lobule VI & $\mathrm{L}$ & 236 & -26 & -50 & -24 & 8.6 & 482 & -22 & -52 & -24 & 11.2 \\
\hline Cerebellum: Lobule VIIb & $\mathrm{L}$ & 43 & -12 & -70 & -46 & 7.5 & 48 & -14 & -68 & -46 & 7.9 \\
\hline Cerebellum: Lobule VIIIa/b & $\mathrm{L}$ & 51 & -20 & -58 & -48 & 6.4 & 165 & -26 & -58 & -54 & 8.8 \\
\hline Thalamus & $\mathrm{L}$ & & & & & & 81 & -16 & -22 & 2 & 10.4 \\
\hline Middle Temporal Gyrus (37) & $\mathrm{L}$ & & & & & & 255 & -44 & -70 & 0 & 9.1 \\
\hline Middle Temporal Gyrus (37) & $\mathrm{R}$ & & & & & & 30 & 44 & -58 & 12 & 7.0 \\
\hline Anterior Insula (13) & $\mathrm{L}$ & & & & & & 31 & -32 & 14 & 8 & 8.1 \\
\hline Anterior Insula (13) & $\mathrm{R}$ & & & & & & 10 & 32 & 16 & 6 & 6.5 \\
\hline Putamen & $\mathrm{R}$ & & & & & & 47 & 24 & 2 & 8 & 8.0 \\
\hline Putamen & $\mathrm{L}$ & & & & & & 52 & -22 & 0 & 10 & 7.8 \\
\hline Precuneus (31) & $\mathrm{L}$ & & & & & & 32 & -28 & -72 & 24 & 7.1 \\
\hline
\end{tabular}

$\boldsymbol{B} \boldsymbol{A}=$ Brodmann Area $; \boldsymbol{K}_{\boldsymbol{E}}=$ cluster size; $\boldsymbol{x}, \boldsymbol{y}, \boldsymbol{z}=$ MNI coordinates at cluster peak; $\boldsymbol{t}_{\boldsymbol{m a x}}=t$-statistic at cluster peak; All results are whole-brain voxel-corrected $p_{F W E}<0.05(t>5.7)$. 
Table S2: Group Differences and Clinical Correlations

\begin{tabular}{|c|c|c|c|c|c|c|}
\hline Region (BA) & & $K_{E}$ & $x$ & $y$ & $z$ & $t_{\max }$ \\
\hline \multicolumn{7}{|l|}{ Self-Paced: HC $>$ FRDA } \\
\hline Cerebellum: Lobule VI & $\mathrm{R}$ & 230 & 28 & -58 & -24 & 3.73 \\
\hline \multicolumn{7}{|l|}{ Self-Paced: FARS Correlations (Negative) } \\
\hline Pre-/Supplementary Motor Area (6) & - & 226 & 6 & 2 & 64 & 4.70 \\
\hline Inferior Parietal Lobule (40) & $\mathrm{L}$ & 214 & -50 & -44 & 24 & 4.14 \\
\hline Anterior Insula (13) & $\mathrm{L}$ & 262 & -40 & 24 & 2 & 4.12 \\
\hline Superior Temporal Gyrus (21) & $\mathrm{L}$ & 281 & -52 & -22 & 0 & 4.04 \\
\hline \multicolumn{7}{|l|}{ Cued Multi-Finger: FRDA $>$ HC } \\
\hline Precuneus (7) & - & 1117 & -8 & -48 & 70 & 5.30 \\
\hline Intraparietal Sulcus (40) & $\mathrm{R}$ & 605 & 46 & -36 & 56 & 4.85 \\
\hline Intraparietal Sulcus (40) & $\mathrm{L}$ & 416 & -36 & -42 & 52 & 4.02 \\
\hline Dorsolateral Prefrontal Cortex (9) & $\mathrm{R}$ & 898 & 48 & 32 & 28 & 4.57 \\
\hline Ventral Premotor Cortex (6) & & & 50 & 12 & 26 & 4.52 \\
\hline Dorsolateral Prefrontal Cortex (9) & $\mathrm{L}$ & 400 & -38 & 26 & 26 & 3.98 \\
\hline Ventral Premotor Area (6) & & & -56 & 14 & 30 & 3.81 \\
\hline Fusiform Gyrus (37) & $\mathrm{R}$ & 361 & 54 & -64 & -14 & 4.74 \\
\hline Caudate & $\mathrm{R}$ & 544 & 22 & -26 & 18 & 4.56 \\
\hline Caudate & $\mathrm{L}$ & 589 & -18 & -16 & 22 & 5.22 \\
\hline Cerebellum: Crus I & $\mathrm{L}$ & 929 & -38 & -64 & -36 & 4.12 \\
\hline Cerebellum: Vermis Crus II & & & 4 & -78 & -32 & 4.01 \\
\hline
\end{tabular}

$\boldsymbol{B} \boldsymbol{A}=$ Brodmann Area; $\boldsymbol{K}_{\boldsymbol{E}}=$ cluster size; $\boldsymbol{x}, \boldsymbol{y}, \boldsymbol{z}=$ MNI coordinates at cluster peak; $\boldsymbol{t}_{\boldsymbol{m a x}}=$ $t$-statistic at cluster peak; All results are cluster-corrected $p_{F W E}<0.05(t>2.4$, cluster extent

$$
>\text { 213) }
$$

Table S3: Between-group fMRI activation differences in controls versus FRDA subgroup during self-paced single finger tapping

\begin{tabular}{|c|c|c|c|c|c|c|}
\hline \multicolumn{7}{|c|}{ during self-paced single finger tapping } \\
\hline & \multicolumn{3}{|c|}{$\underline{\text { FARS }}_{\text {LOW }}>$ CONTROLS } & \multicolumn{3}{|c|}{$\underline{\text { FARS }}_{\underline{H I G H} \leq \text { CONTROLS }}$} \\
\hline ROI & $T_{\max }$ & $K e$ & $p_{\text {corrected }}$ & $T_{\max }$ & Ke & $p_{\text {corrected }}$ \\
\hline aIns & 2.31 & 33 & 0.064 & 4.07 & 129 & $<0.001$ \\
\hline STG & 3.49 & 108 & $<0.001$ & 2.85 & 59 & 0.011 \\
\hline$\overline{\mathrm{IPL}}$ & 3.26 & 155 & $<0.001$ & 3.65 & 19 & 0.17 \\
\hline SMA & 3.06 & 42 & 0.036 & 2.60 & 37 & 0.049 \\
\hline
\end{tabular}

Results are cluster-corrected FWE $p<0.05$ within the mask of FARS-correlated brain regions (Figure 2), using a voxel-level cluster-forming threshold of $p<0.05$. ROI, region-of-interest; $\boldsymbol{T}_{\max }$, peak $T$-value within the cluster;

$\boldsymbol{K}_{\boldsymbol{E}}$, cluster size; $\boldsymbol{p}_{\text {corrected }}$, cluster-level significance 
Table S4: Group Difference Effect Sizes (Cohen's d) with and without late-onset FRDA (LOFA)

\begin{tabular}{|c|c|c|c|}
\hline \\
\hline \multicolumn{2}{|l|}{ Region (BA) } & \multirow[t]{2}{*}{$A L L$} & $\begin{array}{c}\text { No } \\
L O F A \\
\end{array}$ \\
\hline \multicolumn{3}{|l|}{ Self-Paced: HC > FRDA } & \\
\hline Cerebellum: Lobule VI & & 0.67 & 0.65 \\
\hline \multicolumn{4}{|l|}{ Cued Multi-Finger: FRDA > HC } \\
\hline Precuneus (7) & - & 1.14 & 0.97 \\
\hline Intraparietal Sulcus (40) & $\mathrm{L}$ & 1.22 & 1.03 \\
\hline Intraparietal Sulcus (40) & $\mathrm{R}$ & 1.52 & 1.36 \\
\hline Dorsolateral Prefrontal Cortex (9) & $\mathrm{L}$ & 1.36 & 1.23 \\
\hline Dorsolateral Prefrontal Cortex (9) & $\mathrm{R}$ & 1.47 & 1.59 \\
\hline Fusiform Gyrus (37) & $\mathrm{R}$ & 1.17 & 1.16 \\
\hline Caudate & $\mathrm{L}$ & 1.43 & 1.25 \\
\hline Caudate & $\mathrm{R}$ & 1.17 & 1.04 \\
\hline Cerebellum: Crus I & $\mathrm{L}$ & 1.11 & 1.10 \\
\hline $\begin{array}{r}A L L=33 \text { controls, } 25 \text { FRDA } \\
\text { (excluding } 6 \text { FRDA with }\end{array}$ & & $\begin{array}{l}\text { itrols, } \\
\text { vears }\end{array}$ & FRDA \\
\hline
\end{tabular}




\section{Cerebral Compensation during Motor Function in Friedreich Ataxia: The IMAGE- FRDA Study}

Ian H. Harding, $\mathrm{PhD}^{1}$, Louise A. Corben, $\mathrm{PhD}^{1,2}$, Martin B. Delatycki, $\mathrm{PhD}^{1,2,3}$, Monique R. Stagnitti, BSc(Hons) ${ }^{1}$, Elsdon Storey, $\mathrm{PhD}^{4}$, Gary F. Egan, $\mathrm{PhD}^{1,5}$, Nellie GeorgiouKaristianis, $\mathrm{PhD}^{1}$

${ }^{1}$ School of Psychological Sciences \& Monash Institute of Cognitive and Clinical Neurosciences, Monash University, Melbourne, Australia ${ }^{2}$ Bruce Lefroy Centre, Murdoch Childrens Research Institute, Melbourne, Australia ${ }^{3}$ Clinical Genetics, Austin Health, Melbourne, Australia ${ }^{4}$ Department of Medicine, Monash University, Melbourne, Australia ${ }^{5}$ Monash Biomedical Imaging, Monash University, Melbourne, Australia

Correspondence:

Dr. Ian Harding

School of Psychological Sciences

18 Innovation Walk

Monash University, VIC, 3800

Australia

Email: ian.harding@monash.edu

Phone: +61-3-9905-9283

Fax: +61-3-9905-3948

Running Title: Compensation in Friedreich Ataxia

Word Count: 3783; Figures: 4; Tables: 1; Supplementary Materials: 2 Figures, 4 Tables

Disclosures: The authors report no conflicts of interest

Funding: Australian National Health and Medical Research Council (Project Grant 1046037; Fellowship 1106533)

Keywords: Friedreich ataxia, compensation, fMRI, motor function 


\section{Abstract}

Background: Friedreich ataxia is characterized by progressive motor incoordination that is linked to peripheral, spinal, and cerebellar neuropathy. Cerebral abnormalities are also reported in Friedreich ataxia, but their role in disease expression remains unclear.

Methods: In this cross-sectional functional magnetic resonance imaging study, 25 individuals with Friedreich ataxia and 33 healthy controls performed simple (self-paced single-finger) and complex (visually-cued multi-finger) tapping tasks to respectively gauge basic and attentionally-demanding motor behaviour. For each task, whole brain functional activations were compared between groups, and correlated with disease severity and offline measures of motor dexterity.

Results: During self-pacedsimple finger tapping, cerebral hyperactivation in individuals with Friedreich ataxia at the lower end of clinical severity and cerebral hypoactivation in those more severely affected was observed in premotor/ventral attention brain regions, including the supplementary motor area and anterior insula. Greater activation in this network correlated with greater offline finger tapping precision. Complex, aAttentionally_-demanding_, externally etedfinger tapping was also associated with cerebral hyperactivation, but in this case within dorsolateral prefrontal regions of the executive control network and superior parietal regions of the dorsal attention system. Greater offline motor precision was associated with less activation in the dorsal attention network.

Discussion: Compensatory activity is evident in the cerebral cortex in individuals with Friedreich ataxia. Early compensation followed by later decline in premotor/ventral attention systems demonstrates capacity-limited neural reserve, while the additional engagement of higher-order brain networks is indicative of compensatory task strategies. Network-level changes in cerebral brain function thus potentially serve to mitigate the impact of motor impairments in Friedreich ataxia. 


\section{Introduction}

Friedreich ataxia (FRDA), the most common inherited ataxia, is an autosomal recessive disorder defined by progressive movement incoordination. The neurological component of FRDA includes degeneration of the dorsal root ganglia, dorsal spinal tracts, and dentate nuclei

of the cerebellum ${ }^{1}$. Disrupted functional and structural connectivity between the cerebellum and cerebrum have also been reported ${ }^{2,3}$, as have functional and structural abnormalities in the cerebellar cortex, cerebral cortex, and subcortical extrapyramidal system ${ }^{4-8}$. The emerging picture of the central neuropathology underlying FRDA is thus now expanding beyond conventional focus on sub-tentorial disruptions; however, the role of the cerebrum in disease expression is not well understood.

Motor deficits are the most salient clinical features of FRDA, and include limb and gait ataxia; poor balance control; dysmetria, dysdiadochokinesia, and intention tremor; and dysarthria and dysphagia ${ }^{9}$. The neurofunctional correlates of motor dysfunction have been investigated in vivo using functional magnetic resonance imaging (fMRI) in small cohorts of between 7 and 11 individuals with FRDA ${ }^{10,11}$. These studies found consistent evidence of functional deficits in cerebellar lobules V-VI, the supplementary motor areas, and the inferior parietal lobules during various finger and hand tapping tasks. Other frontal, prefrontal, posterior parietal and cerebellar brain regions were more inconsistently implicated, and observations included both hypoactivation and hyperactivation relative to healthy controls $^{10,11}$. This pattern of findings suggests that a common set of brain abnormalities may contribute to the motor deficits in FRDA, while task-specific mechanisms of functional compensation $^{12}$ or down-stream consequence (i.e., diaschisis ${ }^{13}$ ) may operate elsewhere. 
The current study had two primary aims. First, we sought to replicate and extend upon investigations of simple motor functions in FRDA $^{10,11}$ using a larger research cohort, providing for increased statistical power and more reliable generalizability of results.

Additionally, by recruiting individuals across a wide range of clinical severity, we sought to cross-sectionally investigate links between disease severity and detrimental or compensatory functional changes in the brain.

Second, we aimed to investigate complex motor-related function in FRDA by employing an attentionally demanding motor paradigm. Adaptive real-world interactions rely not just on the ability to produce motor responses, but to do so in a goal-oriented and contextually relevant fashion. Behavioral studies of FRDA indicate deficits not only in motor execution, but also in motor planning and adaptation of motor output in response to changing contextual conditions ${ }^{14,15}$. These results indicate that neural abnormalities may manifest at the interface between cognitive and motor processes within the cerebral cortex ${ }^{16}$. Moreover, greater task demands may also emphasize underlying functional brain deficits, or challenge the limits of active compensatory processes ${ }^{17}$. Taken together, investigating attentionally demanding motor production may more closely approximate real-world behavioral demands and provide new insights into the role the cerebrum may play in the morbidity of FRDA.

In both task manipulations, we predicted that functional hypoactivation would manifest in core motor-related brain regions that have previously been associated with FRDA, including lobules V-VI of the cerebellum, the supplementary motor areas, and the inferior parietal lobules. Compensatory hyperactivation was expected in alternate networks, encompassing the premotor and prefrontal cortices, which are engaged to support neural deficiencies in primary task $\operatorname{areas}^{17,18}$. 


\section{Materials and Methods}

\section{Participants}

Data from 25 individuals with FRDA, homozygous for a GAA expansion in intron 1 of $F X N$, and 33 age and gender-matched control participants without neurological symptoms were available for analysis (Table 1). A further 8 FRDA and 4 control datasets were excluded after data collection due to in-scanner motion or sleep, incorrect task performance, or data corruption (see Supplementary Materials). Six individuals met criteria for late-onset FRDA ( $>25$ years old); supplementary analyses showed that between-group differences were not driven or suppressed by this subgroup (Table S4). All participants were right-handed with the

exception of 1 FRDA. The data was collected as part of the IMAGE-FRDA study ${ }^{4-6}$ (see Supplementary Materials). This project is sanctioned by the Monash Health Human Research Ethics Committee; all participants gave informed, written consent.

\section{$<<$ TABLE $1>>$}

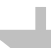

$\sqrt{10}$

Offline Motor Tasks

Participants performed speeded and paced finger tapping tasks ${ }^{19}$. During speeded tapping, participants pressed a button with their non-dominant index finger as rapidly as possible for 10s, followed by an approximate 10s rest period, repeated five times (Figure S1A). Average tapping rate $(\mathrm{Hz})$ interval was recorded (Table 1). During paced tapping, participants alternated between pressing two buttons, one with each thumb, in time with a repeated tone presented every $550 \mathrm{~ms}(1.8 \mathrm{~Hz})$. After 11 taps, the tone ceased and participants were required to maintain the same pace for an additional 31 taps. This procedure was repeated five times over approximately 3mins (Figure S1B). Tapping precision was calculated as the inverse of 
the standard deviation of the difference between observed and correct inter-tap intervals

\section{(Table 1).}

Online Behavioral Stimuli and fMRI Task Design

During fMRI recording, two experimental motor paradigms were performed: a self-paced regularly-timed single-finger tapping paradigm $(\mathrm{SF})^{10}$, and a visually-cued irregularly-timed multi-finger tapping paradigm (MF); Figure S1. Each paradigm started and ended with a 16s rest period, and consisted of four $24 \mathrm{~s}$ task blocks interspersed by $16 \mathrm{~s}$ rest blocks (176s total per task). During each task, an image of the palmar aspect of a right hand with fingers abducted was shown on a black background. During the SF task, a green circle appeared on the index finger continuously for the 24s duration of the block (Figure S1C). With this cue, participants were asked to tap their index finger and thumb together at $0.67 \mathrm{~Hz}$, a rate at which they were trained immediately prior to data collection (while in the scanner). Average tapping rate was recorded. During the MF task blocks, the small green circle appeared in a random order on the index, middle, ring or little finger for $300 \mathrm{~ms}$, with a varied interstimulus interval averaging $1200 \mathrm{~ms}$ (varied at 550ms, 940ms, or 2240ms; Figure S1D). Tapping accuracy, defined as the percentage of trials in which the indicated finger was correctly tapped to the thumb, was recorded. The order of SF and MF task presentation Task order was counter-balanced between across participants.

Stimuli were presented using E-prime (Psychological Software Tools, Pittsburgh, USA). A MRI-compatible right-handed glove with movement sensors (Fifth Dimension Technology, www.5DT.com) was used to record the kinematics of the finger movements. During the prescan practice session, the kinematic characteristics (range of each finger movement) were calibrated for each individual. 


\section{MRI data acquisition and preprocessing}

All participants were scanned using a 3 Tesla Siemens Skyra scanner (Siemens, Erlangen, Germany) with a 32-channel head coil at Monash Biomedical Imaging, Victoria, Australia. The functional run consisted of 157 whole brain, gradient-echo echo-planar images (GREEPIs) comprising 44 interleaved, contiguous axial slices $\left(\mathrm{TR}=2500 \mathrm{~ms}\right.$; $\mathrm{TE}=30 \mathrm{~ms}$; flip $=90^{\circ}$; $3 \mathrm{~mm}$ isotropic voxels; FOV=192 $\times 192 \mathrm{~mm})$. A whole-brain T1-weighted magnetizationprepared rapid gradient-echo (MPRAGE) structural image was also acquired for each participant (176 sagittal slices; $1.0 \mathrm{~mm}$ isotropic voxels; TR=1900ms; TE=2.19ms; $\mathrm{FOV}=256 \times 256 \mathrm{~mm})$.

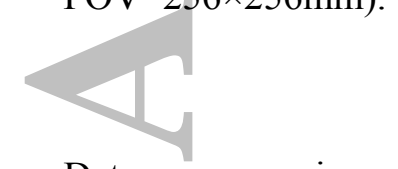

Data preprocessing using SPM12 (Functional Imaging Laboratory, UCL, UK;

www.fil.ion.ucl.ac.uk/spm/) consisted of: (i) temporal registration to the middle slice of each functional volume; (ii) spatial alignment to the first volume of the run; (iii) spatial unwarping to account for susceptibility-by-movement noise variance; (iii) coregistration with the T1weighted structural image; (iv) skull-stripping, segmentation, and non-linear normalization of the T1 (and co-registered EPIs) to standard anatomical space (MNI); and (v) spatial smoothing using a Gaussian kernel of $5 \mathrm{~mm}$ full-width at half-maximum (FWHM).

\section{Data Analysis: Group Activation Differences}

Task fMRI effects were quantified using hierarchical general linear modeling. At the individual level, the task conditions (SF and MF) were coded as block predictors, convolved with a canonical hemodynamic response function. Additional regressors were included in the

GLM to robustly account for motion-related variance (3 planes of translation; 3 axes of rotation; and their first derivatives) and physiological noise (5 principal components of the fMRI signal in the white matter and cerebrospinal fluid, i.e., $\operatorname{CompCor}^{20}$ ). 
Contrast estimates of the individual-level SF and MF effects were entered into group-level two-sample t-tests to infer population differences across the whole brain, while controlling for age, gender, and task performance. Separate group-level models were estimated for the SF and MF task effects. Homogeneity of variance between groups was not assumed; heteroscedasticity was instead estimated using a standard ReML approach implemented in SPM12 and the statistics and degrees of freedom were adjusted as appropriate (analogous to Welch's t-test). Statistical thresholds were corrected for multiple comparisons (cluster-based family-wise error corrected $\mathrm{p}<0.05$ ) using non-parametric Monte Carlo simulations.

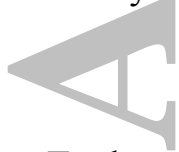

To determine whether significant between-group activation differences were associated with brain atrophy, group comparisons (two-sample t-tests) of grey matter volume (assessed by voxel-based morphometry ${ }^{5}$ ) and function-structure correlations were assessed (Supplementary Materials).

\section{Data Analysis: Clinical Correlations}

Linear relationships between task activations and disease severity across the whole brain were assessed using multiple regression models with Friedreich Ataxia Rating Scale (FARS; higher scores indicates more severe disease $\mathrm{e}^{21}$ ) score as the predictor variable and age, gender, and task performance as nuisance covariates. Regressions modeling the GAA triplet-repeat expansion on the shorter $F X N$ allele (GAA1) were also assessed, controlling for FARS score.

Further exploration and characterization of significant clinical effects was undertaken using a post-hoc median split. The FRDA sample was divided into two sub-groups based on disease 
severity $\left(\mathrm{n}_{\text {low }}=12\right.$, mean $\mathrm{FARS}_{\text {low }}=58 ; \mathrm{n}_{\text {high }}=13$, mean $\left.\mathrm{FARS}_{\text {high }}=95\right)$, and planned post -hoc comparisons were undertaken between each clinical sub-group and the healthy control cohort.

\section{Brain-Behaviour Correlations}

Activity within disease-sensitive functional regions (i.e., between-group differences or clinical correlations) was further assessed for correlations with offline behaviouralmotor performance measures. As detailed below, when overlaid onto an atlas of well-described functional brain networks ${ }^{22,23}$, the observed task-related functional abnormalities mapped selectively onto different networks. SP and MF task-related activations were averaged across disease-sensitive regions falling within each network, and correlated with offline motor behavior (speeded motor performance and paced motor precision).

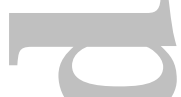

\section{Results}

\section{Finger-Tapping Behavior}

Offline maximal tapping rate and tapping precision were significantly slower in individuals with FRDA relative to controls (both $\mathrm{p}<0.001$; Table 1). Online self-paced tapping rate was significantly greater in the FRDA cohort $(\mathrm{p}=0.022)$, while multi-finger accuracy was matched ( $p=0.90$, although a majority of participants were operating at ceiling; Table 1).

\section{Self-Paced, Single Finger Brain Activations}

During self-paced single finger tapping, both cohorts engaged the left primary motor (M1) and somatosensory cortices (S1), midline supplementary motor area (SMA), right inferior parietal lobule (IPL), and right cerebellar lobule V (Figure 1; Table S1). Controls additionally showed evidence of significant activation in the right and left ventral premotor cortex, left insula, and right cerebellar lobule VIIIb. 
$<<$ FIGURE 1 $>>$

Relative to the control cohort, individuals with FRDA had significantly reduced activations in right lobule VI of the cerebellum (Figure 2; Table S2); no significant activation increases were evident. Functional abnormalities in lobule VI were not significantly associated with brain atrophy (Supplementary Materials). A supplementary subgroup analysis confirmed that group differences in tapping rate were not driving this effect (Supplementary Materials).

In the FRDA cohort, greater disease severity (FARS score) was associated with lesser activation in the supplementary motor area (SMA), left anterior insula (aIns), left inferior parietal lobule (IPL), and left superior temporal gyrus (STG) (Figure 2; Table S2). No significant associations with GAA1 (controlling for FARS) were evident.

As illustrated in Figure 2, post-hoc median-split comparisons revealed, relative to healthy controls, significantly increased activations in the FARS $\mathrm{S}_{\mathrm{low}}$ subgroup and decreased

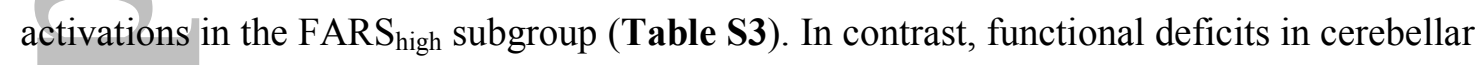
lobule VI showed monotonic decline from healthy to $\mathrm{FARS}_{\text {low }}$ to $\mathrm{FARS}_{\text {high. }}$

$<<$ FIGURE 2>>

\section{Visually Cued, Multi-Finger Brain Activations}

Cued multi-finger tapping was associated with activations in both cohorts bilaterally in the motor system (M1, S1, SMA, dorsal and ventral premotor), intraparietal parietal sulci (IPS), inferior and superior parietal lobules, caudate nuclei, and cerebellar lobules V-VII and VIIbVIIIb. Additionally activation was observed in the FRDA cohort in the dorsolateral prefrontal 
cortex (dlPFC) bilaterally, the dorsal precuneus, and posterior aspects of the right middle temporal gyrus (Figure 1; Table S1).

Relative to healthy controls, individuals with FRDA elicited greater activation in bilateral dIPFC, ventrolateralal premotor areas, IPS, and caudate nuclei, as well as the medial precuneus, right fusiform gyrus, and left cerebellar crus I (Figure 3; Table S2). These functional abnormalities were not significantly associated with brain atrophy (Supplementary Materials).

There was no evidence of FARS correlations at whole-brain corrected levels, but within the mask of regions demonstrating between-group activation differences, positive associations were found in the precuneus $\left(t_{\max }=2.71 ; \mathrm{K}_{\mathrm{E}}=88 ; \mathrm{p}_{\text {corrected }}=0.033\right)$ and right IPS $\left(t_{\max }=3.47\right.$; $\left.\mathrm{K}_{\mathrm{E}}=94 ; \mathrm{p}_{\text {corrected }}=0.025\right)$. Greater GAA1 was associated with less task-related activation in the anterior cingulate cortex $\left(t_{\max }=5.05 ; \mathrm{K}_{\mathrm{E}}=156 ; \mathrm{p}_{\text {corrected }}<0.001\right)$, right temporal-parietal junction $\left(t_{\max }=5.48 ; \mathrm{K}_{\mathrm{E}}=70 ; \mathrm{p}_{\text {corrected }}=0.044\right)$, and left lateral precuneus $\left(t_{\max }=5.09 ; \mathrm{K}_{\mathrm{E}}=70\right.$;

$\left.\mathrm{p}_{\text {corrected }}=0.044\right)$; Figure S2.

Post-hoc median-split analysis revealed several activation profiles (Figure 3). First, the bilateral dIPFC are not responsive to task demands in healthy controls, but are engaged in FRDA with no statistical differences between greater and lesser disease severity. Second, the precuneus is also not engaged in controls, but shows localized parametric increases in activation as disease severity increases. Finally, the bilateral IPS are active in controls, but this activity is increased in FRDA and a significant positive relationship with disease severity is observed in the right hemisphere. 


\section{Network-Level Brain-Behaviour Correlations}

Based on an atlas of intrinsic functional human brain networks ${ }^{22,23}$, it was observed that all brain regions that were sensitive to FRDA disease status during single-finger tapping reside

within the salience (also known as the ventral attention) network, except the superior temporal gyrus (Figure 4A). In the FRDA cohort, greater average functional activations within ventral attention regions was associated with better performance on the offline motor precision task (Spearman's $\rho=0.40, p=0.05)$, but not speeded tapping performance $(\rho=0.16$, $\mathrm{p}=0.45)$.

Similarly, all areas of statistically greater activation in the FRDA cohort during multi-finger tapping were found in the dorsal attention (Figure 4B) and executive control (Figure 4C) networks. Greater activation within regions overlapping the dorsal attention network was associated with poorer offline motor precision $(\rho=-0.65, p=0.001)$ and slower speeded tapping $(p=-0.41, p$ $(\rho=-0.41, p=0.044)$ in the FRDA cohort. Activity within the executive control network did not map significantly onto offline motor precision $(\rho=-0.31, p=0.14)$ or speeded tapping $(\rho=-0.21$, $\mathrm{p}=0.32)$.

$<<$ FIGURE $4>>$

\section{Discussion}

In individuals with FRDA, we report functional abnormalities in the cerebral cortex underlying simple self-paced, and more complex attentionally-demanding motor tasks. In the case of simple self-generated motor function, functional changes preferentially impact ventral attention (i.e., 'salience') and premotor systems. Importantly, these changes are sensitive to 
disease status, with increased activation relative to healthy controls evident in less severe FRDA, decreased activation evident in more severe disease states, and correlations between activation magnitude and motor dexterity. On the other hand, attentionally demanding motor function increased engagement of executive control and dorsal attention systems in FRDA, with activation in the latter correlated with motor performance measures. These observations are consistent with models of functional compensation operating at the level of large-scale neural networks, which may serve to mitigate disease-related dysfunction.

Models of functional compensation, although diverse in their specifics, share the premise that degeneration of neural tissues leads to a loss of functional efficiency or capacity ${ }^{17,18,24,25}$. In response, additional neural resources may be recruited to account for neuronal damage and mitigate subsequent behavioral consequences. These compensatory mechanisms may either involve increased activation in affected task networks (i.e., neural reserve), or recruitment of alternative neural regions to complement compromised function (i.e., neural compensation $)^{24,25}$. In the current study, we observed evidence of both neural reserve and neural compensation in the cerebrum in individuals with FRDA.

Structural, functional, and connectivity abnormalities have all been previously reported in areas of the premotor and ventral attention network, including the SMA and anterior insula, in FRDA $^{4,10,26}$. However, in the current case, an inverted-U pattern of functional activation across different levels of disease severity was observed during simple finger tapping. This activation pattern is characteristic of a compensation model whereby increased neural activity is thought to mitigate functional disruptions early in the course of the disease, but which cannot be sustained with continued progression of the underlying pathology ${ }^{12,17,27}$. Similar effects have also been observed across a number other neurodegenerative disorders, including 
Alzheimer disease, Huntington disease, and multiple sclerosis ${ }^{12,28-31}$. This interpretation is further supported in the current experiment by correlations showing that individuals with greater activation in this network also have greater motor precision, providing evidence of socalled "successful compensation"12.

These non-linear cerebral functional changes occurred alongside progressive activation decreases in functionally connected regions of the cerebellum, putatively reflecting primary cerebellar pathology concurrent with secondary mechanisms of cerebral compensation. Indeed, cerebellar changes reported in FRDA are often more strongly linked to disease expression than cerebral measures ${ }^{4,7}$. Early degeneration and dysfunction in the cerebellum may therefore be initially offset by a functional shift to relatively spared, inter-connected areas of the cerebrum. Continued cerebellar pathology, however, may eventually overwhelm the capacity limits of cerebral function, leading to dysfunction across the whole network.

The simple motor task used in this experiment was identical to that employed by Akhlaghi and colleagues (2012) in a smaller sample of 11 individuals with FRDA. In contrast to the current study, those authors reported functional deficits in cerebral premotor and supplementary motor, inferior parietal, and insula/frontal opercular regions (i.e., areas of the ventral attention network), but did not find evidence of hyperactivations. However, the clinical severity of the study sample in this earlier work lies predominantly in the more severe half of the current FARS median split. When the results from Akhlaghi et al (2012) are

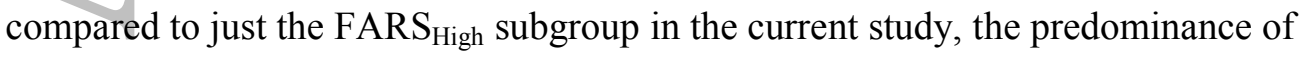
hypoactivations in these regions is replicated. Deficits in lobule V/VI and the SMA were also implicated by Ginestroni and colleagues (2011) during hand tapping. Taken together, functional changes are consistently evident in ventral attention/premotor brain regions during 
motor execution in individuals with FRDA. But importantly, the current study indicates that disease state is an important determinant of a non-linear activation profile across individuals in these regions.

During complex, attentionally demanding motor performance, neural compensation through engagement of alternative functional networks was also readily apparent. In response to task demands, individuals with FRDA activated the executive control network (bilateral dlPFC and cerebellar crus I), and activated or significantly upregulated regions of the dorsal attention network (ventrolateralal premotor areas; IPS; and precuneus). Deficits in functions

traditionally ascribed to these brain areas, including cognitive control and attention, have been reported in $\mathrm{FRDA}^{8,32-34}$, but are generally considered to be subtle aspects of the disorder ${ }^{1}$. Moreover, previous functional MRI studies of cognition in FRDA have generally found a relative sparing of top-down control/attention networks ${ }^{4,8,26}$. As such, individuals with FRDA may utilize these relatively intact executive resources to compensate for impairments in other systems, perhaps by employing different cognitive strategies relative to healthy individuals to successfully mediate behavioral demands ${ }^{18,24,35}$. This shift to alternate strategies is supported by the observation of increased engagement of the dorsal attention network in individuals with FRDA who successfully completed the fMRI task, but who demonstrated worse motor precision using an offline measure. That is, individuals with greater motor impairment relied on higher-order, top-down attentional control systems to a greater degree when undertaking an attentionally-demanding behavioural task.

In contrast to simple motor performance, however, we do not find evidence of compensation failure in individuals with more severe disease severity during the attentionally demanding task. In fact, in subregions of the precuneus and right IPS, there is evidence of continued 
increases in activation with increased disease severity. These findings perhaps again provide evidence for the relative sparing of executive systems in FRDA, allowing neural compensation to remain effective regardless of disease status, at least within the constraints of this task. Future experiments using parametric modulations of cognitive load will be necessary to test the limits of these mechanisms ${ }^{12}$.

Neural deficits were also not apparent during attentionally demanding task performance. This finding is particularly surprising with respect to the cerebellar cortex (which was also explored at more liberal uncorrected statistical thresholds), given that deficits in lobules $\mathrm{V}$ and VI are relatively consistent in structural and functional neuroimaging studies of FRDA $^{4,7,10,11,26}$. However, that we find cerebellar deficits in our simple motor task in the same group of participants, during the same scan session, using similar perceptual stimuli, and employing identical data processing and statistical modeling approaches provides some protection against type-II error. While definitive explanations for this intact cerebellar activation are beyond the scope of the current data, there are several compelling possibilities. First, it may be that the functional reorganization of neural processing from impaired ventral attention systems (including lobule VI) to intact executive systems prevents the former from becoming overloaded ${ }^{35}$. Alternatively, inputs down the rostral-caudal prefrontal hierarchy from higher-order executive to lower-order lateral premotor areas ${ }^{36}$, and subsequently through premotor and motor cortico-cerebellar loops ${ }^{37}$, may sustain task-appropriate neuronal activity. These hypotheses are speculative, but present interesting avenues for future research.

Notably, the simple and complex tasks were selectively sensitive to dysfunction in bottom-up (i.e., salience) and top-down (i.e., executive/dorsal attention) brain networks, respectively. This selectivity may relate to the subjective self-assessment and internal vigilance demands of 
$\underline{\text { the self-paced manipulation, relative to the objective action-outcome evaluation and external }}$ vigilance requirements of the visually-cued task. The current study thus supports dysfunction in both processes in FRDA, the consequences of which may manifest independently in $\underline{\text { different contexts. }}$

Definitive inference of links between cerebral compensation, disease severity, and disease progression are limited by the cross-sectional design of this work. Future longitudinal studies will be necessary to confirm these relationships. Furthermore, due to the technological limitations of fMRI and the behavioral limitations inherent to individuals with FRDA, it was necessary to restrict experimentation of motor function to hand movements (as opposed to, for example, gait impairments or complex real-world behaviours). While the ecological validity of such designs may be questionable, the ability to isolate fundamental dysfunctions is useful in developing more complete neurobiological models of this disorder. Furthermore, while links between brain function and disease severity are reported in this work, investigating the potential modulatory role of differences in age-at-onset ${ }^{34}$ was not possible due to confounds with normal aging processes in this sample (age-at-onset vs. current age: $\mathrm{r}=0.84$ ); further investigations decoupling these effects are warranted.

Contemporary theories, models, and evidence of neural compensation and neural reserve firmly support the notion that neurofunctional changes underlying degenerative processes are not only detrimental, but may also be compensatory ${ }^{12,25,28}$. This idea has driven a groundswell of research into novel therapeutic approaches designed to stimulate or maintain these compensatory cerebral processes through, e.g., non-invasive brain stimulation ${ }^{38,39}$ or cognitive therapies $^{40,41}$. Given the evidence that cerebral compensation may also operate in FRDA, these novel means of intervention represent exciting pathways for future research. 
In summary, the present study motivates a reconceptualization of cerebral contributions to FRDA. In particular, cerebral alterations are likely not merely the secondary detriments of cerebellar and spinal insults. Rather, cerebral changes may represent capacity limited compensatory processes operating at the network level of brain function, which serve to mitigate the behavioral consequences of progressive disease-related pathology. Critically, these novel findings inform development of new avenues of clinical intervention for FRDA.

\section{Acknowledgements}

This study was supported by funding from the Australian National Health and Medical Research Council (Project Grant 1046037; Fellowship 1106533 to IHH). We thank the individuals with Friedreich ataxia and healthy controls who participated in this study. The authors declare no conflicts of interest.

\section{Author Roles}

IHH conducted the statistical analyses and wrote the paper; LAC and MBD conceptualized the project, coordinated participant recruitment, and edited the paper; GFE and NG-K conceptualized the project, consulted on the analysis approach, and edited the paper; ES conceptualized the project and edited the paper; MRS recruited the participants, collected/managed the data, conducted statistical analyses, and edited the paper. 


\section{References}

1. Pandolfo M. Friedreich ataxia. Archives of neurology 2008;65(10):1296-1303.

2. Akhlaghi H, Yu J, Corben L, et al. Cognitive deficits in Friedreich ataxia correlate

with micro-structural changes in dentatorubral tract. Cerebellum (London, England) 2014;13(2):187-198.

3. Zalesky A, Akhlaghi H, Corben LA, et al. Cerebello-cerebral connectivity deficits in Friedreich ataxia. Brain structure \& function 2014;219(3):969-981.

4. Harding IH, Corben LA, Storey E, et al. Fronto-cerebellar dysfunction and dysconnectivity underlying cognition in friedreich ataxia: The IMAGE-FRDA study. Human brain mapping 2016;37(1):338-350.

5. Selvadurai LP, Harding IH, Corben LA, et al. Cerebral and cerebellar grey matter atrophy in Friedreich ataxia: the IMAGE-FRDA study. Journal of neurology 2016.

6. Harding IH, Raniga P, Delatycki MB, et al. Tissue atrophy and elevated iron concentration in the extrapyramidal motor system in Friedreich ataxia: the IMAGE-FRDA study. Journal of neurology, neurosurgery, and psychiatry 2016.

7. Rezende TJ, Silva CB, Yassuda CL, et al. Longitudinal magnetic resonance imaging study shows progressive pyramidal and callosal damage in Friedreich's ataxia. Movement disorders : official journal of the Movement Disorder Society 2016;31(1):70-78.

8. Dogan I, Tinnemann E, Romanzetti S, et al. Cognition in Friedreich's ataxia: a behavioral and multimodal imaging study. Annals of clinical and translational neurology 2016;3(8):572-587.

9. Corben LA, Georgiou-Karistianis N, Bradshaw JL, Evans-Galea MV, Churchyard AJ, Delatycki MB. Characterising the neuropathology and neurobehavioural phenotype in 
Friedreich ataxia: a systematic review. Advances in experimental medicine and biology 2012;769:169-184.

10. Akhlaghi H, Corben L, Georgiou-Karistianis N, et al. A functional MRI study of motor dysfunction in Friedreich's ataxia. Brain research 2012;1471:138-154.

11. Ginestroni A, Diciotti S, Cecchi $\mathrm{P}$, et al. Neurodegeneration in friedreich's ataxia is associated with a mixed activation pattern of the brain. A fMRI study. Human brain mapping 2012;33(8):1780-1791.

12. Scheller E, Minkova L, Leitner M, Kloppel S. Attempted and successful compensation in preclinical and early manifest neurodegeneration - a review of task FMRI studies. Frontiers in psychiatry $2014 ; 5: 132$.

13. Carrera E, Tononi G. Diaschisis: past, present, future. Brain : a journal of neurology 2014;137(Pt 9):2408-2422.

14. Corben LA, Delatycki MB, Bradshaw JL, et al. Impairment in motor reprogramming in Friedreich ataxia reflecting possible cerebellar dysfunction. Journal of neurology 2010;257(5):782-791.

15. Corben LA, Georgiou-Karistianis N, Bradshaw JL, Hocking DR, Churchyard AJ, Delatycki MB. The Fitts task reveals impairments in planning and online control of movement in Friedreich ataxia: reduced cerebellar-cortico connectivity? Neuroscience 2011;192:382-390.

16. Ridderinkhof KR, van den Wildenberg WP, Segalowitz SJ, Carter CS. Neurocognitive mechanisms of cognitive control: the role of prefrontal cortex in action selection, response inhibition, performance monitoring, and reward-based learning. Brain and cognition 2004;56(2):129-140.

17. Reuter-Lorenz PA, Cappell KA. Neurocognitive aging and the compensation hypothesis. Curr Dir Psychol Sci 2008;17(3):177-182. 
18. Park DC, Reuter-Lorenz P. The adaptive brain: aging and neurocognitive scaffolding. Annual review of psychology 2009;60:173-196.

19. Stout JC, Paulsen JS, Queller S, et al. Neurocognitive signs in prodromal Huntington disease. Neuropsychology 2011;25(1):1-14.

20. Behzadi Y, Restom K, Liau J, Liu TT. A component based noise correction method (CompCor) for BOLD and perfusion based fMRI. NeuroImage 2007;37(1):90-101.

21. Subramony SH, May W, Lynch D, et al. Measuring Friedreich ataxia: Interrater reliability of a neurologic rating scale. Neurology 2005;64(7):1261-1262.

22. Buckner RL, Krienen FM, Castellanos A, Diaz JC, Yeo BT. The organization of the human cerebellum estimated by intrinsic functional connectivity. Journal of neurophysiology $2011 ; 106(5): 2322-2345$.

23. Yeo BT, Krienen FM, Sepulcre J, et al. The organization of the human cerebral cortex estimated by intrinsic functional connectivity. Journal of neurophysiology 2011;106(3):11251165.

24. Barulli D, Stern Y. Efficiency, capacity, compensation, maintenance, plasticity: emerging concepts in cognitive reserve. Trends in cognitive sciences 2013;17(10):502-509. 25. Fornito A, Zalesky A, Breakspear M. The connectomics of brain disorders. Nature reviews Neuroscience 2015;16(3):159-172.

26. Georgiou-Karistianis N, Akhlaghi H, Corben LA, et al. Decreased functional brain activation in Friedreich ataxia using the Simon effect task. Brain and cognition 2012;79(3):200-208.

27. Saxena S, Caroni P. Selective neuronal vulnerability in neurodegenerative diseases: from stressor thresholds to degeneration. Neuron 2011;71(1):35-48.

28. Schoonheim MM, Filippi M. Functional plasticity in MS: friend or foe? Neurology 2012;79(14):1418-1419. 
29. Georgiou-Karistianis N, Poudel GR, Dominguez DJ, et al. Functional and connectivity changes during working memory in Huntington's disease: 18 month longitudinal data from the IMAGE-HD study. Brain and cognition 2013;83(1):80-91.

30. Poudel GR, Stout JC, Dominguez DJ, et al. Functional changes during working memory in Huntington's disease: 30-month longitudinal data from the IMAGE-HD study. Brain structure \& function 2015;220(1):501-512.

31. Kloppel S, Draganski B, Siebner HR, Tabrizi SJ, Weiller C, Frackowiak RS.

Functional compensation of motor function in pre-symptomatic Huntington's disease. Brain : a journal of neurology 2009;132(Pt 6):1624-1632.

32. Corben LA, Akhlaghi H, Georgiou-Karistianis N, et al. Impaired inhibition of prepotent motor tendencies in Friedreich ataxia demonstrated by the Simon interference task. Brain and cognition 2011;76(1):140-145.

33. Klopper F, Delatycki MB, Corben LA, Bradshaw JL, Rance G, Georgiou-Karistianis

$\mathrm{N}$. The test of everyday attention reveals significant sustained volitional attention and working memory deficits in friedreich ataxia. Journal of the International Neuropsychological Society: JINS 2011;17(1):196-200.

34. Nachbauer W, Bodner T, Boesch S, et al. Friedreich ataxia: executive control is related to disease onset and GAA repeat length. Cerebellum (London, England) 2014;13(1):9-

\section{6.}

35. Noppeney U, Friston KJ, Price CJ. Degenerate neuronal systems sustaining cognitive functions. Journal of anatomy 2004;205(6):433-442.

36. Badre D. Cognitive control, hierarchy, and the rostro-caudal organization of the frontal lobes. Trends in cognitive sciences 2008;12(5):193-200. 
37. Kelly RM, Strick PL. Cerebellar loops with motor cortex and prefrontal cortex of a nonhuman primate. The Journal of neuroscience : the official journal of the Society for Neuroscience 2003;23(23):8432-8444.

38. Miniussi C, Cappa SF, Cohen LG, et al. Efficacy of repetitive transcranial magnetic stimulation/transcranial direct current stimulation in cognitive neurorehabilitation. Brain stimulation 2008;1(4):326-336.

39. Floel A. tDCS-enhanced motor and cognitive function in neurological diseases.

NeuroImage 2014;85 Pt 3:934-947.

40. Valenzuela M, Sachdev P. Can cognitive exercise prevent the onset of dementia?

Systematic review of randomized clinical trials with longitudinal follow-up. The American journal of geriatric psychiatry : official journal of the American Association for Geriatric Psychiatry 2009;17(3):179-187.

41. Simon SS, Yokomizo JE, Bottino CM. Cognitive intervention in amnestic Mild Cognitive Impairment: a systematic review. Neuroscience and biobehavioral reviews 2012;36(4):1163-1178.

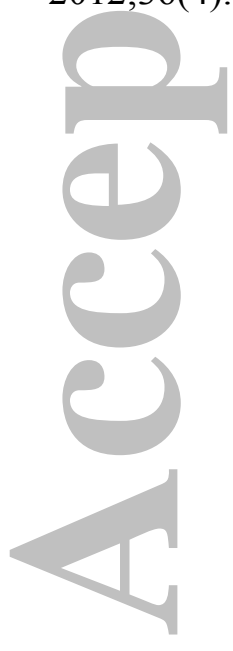




\section{Figure Legends}

Figure 1: Within-Group Task fMRI Activations.

Functional activations during Self-Paced Single Finger (Top) and Cued Multi-Finger (Bottom) Tasks (FWE-corrected $\mathrm{p}_{\text {cluster }}<0.05$ with cluster-forming $\mathrm{p}_{\text {voxel }}<0.001$ ). Activations specific to controls are displayed in red, to FRDA in green, and common to both in yellow.

Figure 2: Between-Group fMRI Activations during Self-Paced Tapping.

Significant group functional differences (FRDA vs. Controls) in the cerebellum (left) and clinical correlations in the cerebrum (right) during self-paced single finger tapping. Bar graphs illustrate mean group fMRI activations $( \pm \mathrm{SD})$, relative to resting baseline, for controls (blue), and a median split of the FRDA group into low disease severity (red), and high disease severity (green) subgroups. FARS, Friedreich Ataxia Rating Scale; STG, superior temporal gyus; IPL, inferior parietal lobule; SMA, supplementary motor area.

Figure 3: Between-Group fMRI Activations during Cued Multi-Finger Tapping. Significant group functional differences (FRDA vs. Controls) in the cerebrum during cued multi-finger tapping. Bar graphs illustrate mean group fMRI activations $( \pm \mathrm{SD})$, relative to resting baseline, for controls (blue), and a median split of the FRDA group into low disease severity (red), and high disease severity (green) subgroups. FARS, Friedreich Ataxia Rating Scale; IPS, intraparietal sulcus; dlPFC, dorsolateral prefrontal cortex.

Figure 4: Network-level Correlations with Offline Motor Behaviour Performance. Linear correlations between offline motor precision and fMRI activation magnitude during self-paced single finger tapping (SP) in disease-relevant brain areas (orange-red; see Figure 2) that overlap with the ventral attention brain network (A; cyan), and fMRI activation magnitude during externally-cued multi-finger tapping (MF) and disease-relevant brain areas (orange-red; see Figure 3) that overlap with the dorsal attention (B; blue) and executive control (C; green) brain networks. Brain network illustration modified from Yeo et al, 2011. Spearman correlation coefficients are reported $(* \mathrm{p}<0.05, * * \mathrm{p}<0.001)$. 


\section{University Library}

\section{- M M N E R VA A gateway to Melbourne's research publications}

Minerva Access is the Institutional Repository of The University of Melbourne

\section{Author/s:}

Harding, IH;Corben, LA;Delatycki, MB;Stagnitti, MR;Storey, E;Egan, GF;Georgiou-Karistianis, $\mathrm{N}$

Title:

Cerebral Compensation During Motor Function in Friedreich Ataxia: The IMAGE-FRDA Study

Date:

2017-08-01

\section{Citation:}

Harding, I. H., Corben, L. A., Delatycki, M. B., Stagnitti, M. R., Storey, E., Egan, G. F. \& Georgiou-Karistianis, N. (2017). Cerebral Compensation During Motor Function in Friedreich Ataxia: The IMAGE-FRDA Study. MOVEMENT DISORDERS, 32 (8), pp.1221-1229. https:// doi.org/10.1002/mds.27023.

Persistent Link:

http://hdl.handle.net/11343/292947 